\title{
Integrated single-cell and bulk RNA sequencing analysis identifies a cancer associated fibroblast-related signature for predicting prognosis and therapeutic responses in colorectal cancer
}

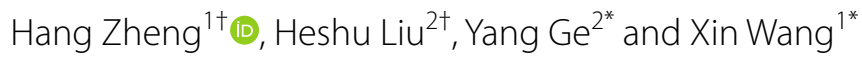

\begin{abstract}
Background: Cancer-associated fibroblasts (CAFs) contribute notably to colorectal cancer (CRC) tumorigenesis, stiffness, angiogenesis, immunosuppression and metastasis, and could serve as a promising therapeutic target. Our purpose was to construct CAF-related prognostic signature for CRC.

Methods: We performed bioinformatics analysis on single-cell transcriptome data derived from Gene Expression Omnibus (GEO) and identified 208 differentially expressed cell markers from fibroblasts cluster. Bulk gene expression data of CRC was obtained from The Cancer Genome Atlas (TCGA) and GEO databases. Univariate Cox regression and least absolute shrinkage operator (LASSO) analyses were performed on TCGA training cohort $(n=308)$ for model construction, and was validated in TCGA validation $(n=133)$, TCGA total $(n=441)$, GSE39582 $(n=470)$ and GSE17536 $(n=177)$ datasets. Microenvironment Cell Populations-counter (MCP-counter) and Estimate the Proportion of Immune and Cancer cells (EPIC) methods were applied to evaluated CAFs infiltrations from bulk gene expression data. Real-time polymerase chain reaction (qPCR) was performed in tissue microarrays containing 80 colon cancer samples to further validate the prognostic value of the CAF model. pRRophetic and Tumor Immune Dysfunction and Exclusion (TIDE) algorithms were utilized to predict chemosensitivity and immunotherapy response. Human Protein Atlas (HPA) databases and immunohistochemistry were used to evaluate the protein expressions.
\end{abstract}

Results: A nine-gene prognostic CAF-related signature was established in training cohort. Kaplan-Meier survival analyses revealed patients with higher CAF risk scores were correlated with adverse prognosis in each cohort. MCPcounter and EPIC results consistently revealed CAFs infiltrations were significantly higher in high CAF risk group. Patients with higher CAF risk scores were more prone to not respond to immunotherapy, but were more sensitive to several conventional chemotherapeutics, suggesting a potential strategy of combining chemotherapy with anti-CAF therapy to improve the efficacy of current T-cell based immunotherapies. Univariate and multivariate Cox regression

\footnotetext{
*Correspondence: interna-1@163.com; wangxin_guo@126.com

${ }^{\dagger}$ Hang Zheng and Heshu Liu contributed equally to this work

${ }^{1}$ Department of General Surgery, Peking University First Hospital, Peking

University, Beijing, People's Republic of China

${ }^{2}$ Department of Oncology, Beijing Chaoyang Hospital, Capital Medical

University, Beijing, People's Republic of China
}

(c) The Author(s) 2021. Open Access This article is licensed under a Creative Commons Attribution 4.0 International License, which permits use, sharing, adaptation, distribution and reproduction in any medium or format, as long as you give appropriate credit to the original author(s) and the source, provide a link to the Creative Commons licence, and indicate if changes were made. The images or other third party material in this article are included in the article's Creative Commons licence, unless indicated otherwise in a credit line to the material. If material is not included in the article's Creative Commons licence and your intended use is not permitted by statutory regulation or exceeds the permitted use, you will need to obtain permission directly from the copyright holder. To view a copy of this licence, visit http://creativecommons.org/licenses/by/4.0/. The Creative Commons Public Domain Dedication waiver (http://creativeco mmons.org/publicdomain/zero/1.0/) applies to the data made available in this article, unless otherwise stated in a credit line to the data. 
analyses verified the CAF model was as an independent prognostic indicator in predicting overall survival, and a CAFbased nomogram was then built for clinical utility in predicting prognosis of CRC.

Conclusion: To conclude, the CAF-related signature could serve as a robust prognostic indicator in CRC, which provides novel genomics evidence for anti-CAF immunotherapeutic strategies.

Keywords: Single-cell RNA-seq, Colorectal cancer, Cancer-associated fibroblasts, Tumor microenvironment, Prognosis

\section{Background}

Colorectal cancer (CRC) is the most commonly diagnosed gastrointestinal cancer and is characterized by exhibiting cancer progression due to therapy resistance $[1-3]$. CRC predominantly originates from dysregulated epithelial cells as well as complex genetic abnormalities and molecular mechanisms $[4,5]$, and evolves by an accomplice called tumor microenvironment (TME), which comprises admixtures of stromal, immune and tumor cells as well as cytokines, chemokines and other extracellular matrix (ECM) acellular components [6]. The reciprocal and dynamic interactions between tumor cells and their surrounding TME play crucial roles in CRC tumorigenesis, progression and metastasis, as well as anticancer efficacy and drug resistance $[7,8]$, and have attracted wide attention in recent years.

As the major TME stromal cellular constituents, cancer-associated fibroblasts (CAFs) were found not only to promote tumorigenesis and enhance the aggressiveness of cancer cells, but also to induce chronic inflammation by producing pro-inflammatory cytokines that are responsible for immune tolerance and tumor metastasis [9-12]. Physiologically, quiescent fibroblasts are functionally activated into myofibroblasts in tissue remodeling conditions like wound healing and fibrosis processes, and are responsible for the integrity and equilibrium of ECM, which provides supportive framework for tissues. Once the remodeling process is completed, myofibroblasts are subsequently dwindling away through apoptosis [13]. Pathologically, CAFs are believed to be highly heterogeneous: genetic alterations in normal fibroblasts, pathological activation mediated by tumor cells [14], as well as additional transdifferentiation of epithelial and mesenchymal cells [15-17] have all been viewed as the origins of CAFs. In an interactional TME network inside the tumor, the "wound" does not heal [18] and the activated CAFs are resistant from apoptosis [19]. Excessive ECM proteins depositions and oncogenic molecules secreted constantly by CAFs are responsible for the invasion, stiffness, angiogenesis, immunosuppression, drug resistance and metastasis of CRCs [20-23]. Therefore, targeting CAFs along with current tumor cell-targeting agents could be promising therapeutic strategies to synergistically counteract CRC progression.
Understanding the heterogeneity and identifying the biomarkers of CAFs could be of great significance for survival prediction and CAF-based therapeutic guidance in CRC. Fibroblast activation protein (FAP), $\alpha$-SMA (ACTA2), platelet derived growth factor receptor- $\beta$ (PDGFRB), caveolin 1 (CAV1) and podoplanin (PDPN) are the generally recognized fibroblasts markers in CRC [20, 24-26]. Among them, FAP is the most appealing therapeutic target owing to its selective expressions in tumor as well as its unique collagenase and gelatinase activities [27, 28]. Preclinical studies have verified the promising effects of eliminating FAP-positive CAFs to increase the recruitments of anti-tumor CD8 $+\mathrm{T}$ cells into tumor stroma, hence rekindling the anti-tumor immunity and suppressing tumor progression [29, 30]. However, no further therapeutic efficacy was observed in metastatic CRC patients treated with anti-FAP inhibitor (sibrotuzumab, talabostat) in the subsequent clinical trials [31, 32]. Hence, more effort is needed for the investigations of innovative therapeutic targets to break the logjam of CAF-mediated tumor progression and immune suppression.

Recently, substantial interest has been aroused around advances in single-cell RNA sequencing (scRNA-seq) technology, which is capable of profiling genes as well as discovering distinct oncogenic cellular populations and associated markers at single-cell resolution [33]. Unequivocally characterizing tumor microenvironmental heterogeneity would facilitate uncovering drug resistance mechanisms and identifying more effective targets for individualized managements [34]. In this work, scRNAseq profiles from Gene Expression Omnibus (GEO) (https://www.ncbi.nlm.nih.gov/geo/) colorectal cancer cells datasets were analyzed to describe the CAF subset and its marker genes. In addition, through integrated bioinformatics transcriptome analyses at both singlecell and bulk levels, we aimed to discover promising CAF-targeting therapeutic hallmarks and design a CAFassociated gene signature to predict drug sensitivity and prognosis for CRC patients.

\section{Methods}

\section{Data source and preprocessing}

The scRNA-seq files (raw unique molecular identifier (UMI) counts based on 10X Genomics technology) 
from five CRC tissues were accessed from GSE132257 [35] via GEO database (https://www.ncbi.nlm.nih.gov/ geo/). We randomly picked four tissues (GSM3855011, GSM3855013, GSM3855017, GSM3855018) as the discovery cohort, and sample GSM3855015 was chosen for hallmark validation. The bulk transcriptome RNAseq data and corresponding clinical data were obtained from The Cancer Genome Atlas rectal adenocarcinoma (TCGA-READ) and colon adenocarcinoma (TCGACOAD) through the UCSC Xena browser (GDC hub) (https://gdc.xenahubs.net) [36]. The batch effects were modified through "ComBat" function of sva $\mathrm{R}$ package [37]. In total, 441 CRC samples with survival information were enrolled and then randomly assigned into TCGA training and TCGA validation cohort with a ratio of 7:3. Additionally, transcriptomic data of 470 CRC samples in GSE39582 and 177 CRC samples in GSE17536 were obtained as the external validation cohorts, expression values were respectively normalized via Robust Multiarray Average (RMA) algorithm, and genes mapped to multiple probes were summarized by their mean values.

\section{Single-cell RNA-seq analysis}

We utilized Seurat R package (version 3.0.2) and applied standard downstream processing for scRNA-seq data (https://github.com/satijalab/seurat) [38]. Genes that detected in less than 3 cells as well as cells with less than 200 detected gene numbers were ruled out, and the mitochondria proportion was limited to less than $20 \%$. Then, LogNormalize method was applied for data normalization. T-distributed stochastic neighbor embedding (t-SNE), a nonlinear dimensionality reduction method, was utilized after principal component analysis (PCA) for unsupervisedly clustering and unbiasedly visualizing cell populations on a two-dimensional map [39]. Subsequently, "FindAllMarkers" function was utilized to identify marker genes of each cluster with the filter value of absolute $\log 2$ fold change $(\mathrm{FC}) \geq 1$ and the minimum cell population fraction in either of the two populations was 0.25 . In addition, the expression pattern of each marker gene among clusters were visualized by applying the "DotPlot" function in Seurat. Afterwards, SingleR package (version 1.0.0) was employed for marker-based celltype annotation [40].

\section{Gene Ontology (GO) and the Kyoto Encyclopedia of Genes and Genomes (KEGG) Analyses}

GO and KEGG pathway functional enrichment analyses were conducted through clusterProfiler $\mathrm{R}$ package (version 3.14.3) to assign various biological processes (BPs), molecular functions (MFs), cellular components (CCs) as well as pathways of identified marker genes in the interested cluster [41], $\mathrm{P}<0.05$ was regarded as statistically enriched.

\section{Construction and validation of an individualized CAF-related prognostic signature}

We designed a prognostic signature across CRC patients by focusing on CAF marker genes, which were identified from the CAF-annotated scRNA-seq cluster. The main endpoint of this research was overall survival (OS), and prognostic CAF-related genes were investigated by univariate Cox regression model in the TCGA training dataset. Genes with $\mathrm{P}<0.1$ in univariate Cox analysis were regarded as candidate prognostic genes. To minimize overfitting risk, we then applied the least absolute shrinkage and selection operator (LASSO) Cox regression model via glmnet $R$ package [42], and the CAF signature was calculated as: $C A F$ risk score $=\Sigma\left(\beta_{i}{ }^{*} \operatorname{Exp}_{\mathrm{i}}\right)$, where $\beta_{\mathrm{i}}$ represented the LASSO coefficient of ith gene, and $\operatorname{Exp}_{i}$ was the ith candidate gene's expression value. Subsequently, patients were classified into high- and low-CAF risk groups by their median CAF scores, and their relationship with OS was evaluated via Kaplan-Meier analysis. We generated heatmaps to visualize the association between CAF risk scores and candidate genes. Similarly, we validated our CAF signature in the TCGA validation and TCGA COAD/READ total cohorts, GSE39582 and GSE17536 external validation cohorts.

\section{Tumor microenvironment infiltration estimation}

The relative infiltrations of 24 immune cell types were quantified via single sample gene set enrichment analysis (ssGSEA) with GSVA R package (version 1.34.0) [43]. The gene set for each immune cell subset was accessed from Bindea et al. [44]. Fibroblasts infiltration levels were quantified through Microenvironment Cell Populationscounter (MCP-counter) [45] and Estimate the Proportion of Immune and Cancer cells (EPIC) [46] algorithms via MCPcounter (version 1.2.0) [45] and immunedeconv (version 2.0.3) [47] R packages. Additionally, we applied estimate $R$ package (version 1.0.13) to calculate the stromal and immune scores, which represents the tumorassociated stromal and immune infiltration levels of each sample [48].

\section{Chemotheraeutic sensitivity and immunotherapy response predictions}

To predict chemosensitivity between high- and low-CAF risk groups, we used pRRophetic $\mathrm{R}$ package (version 0.5) to extrapolate half-maximal inhibitory concentration (IC50) values by building ridge regression model with ten-fold cross-validation $[49,50]$. Several common anticancer drugs (camptothecin, docetaxel, gefitinib, gemcitabine, pazopanib, sunitinib) and their genetic profiles 
were obtained from the largest publicly accessible pharmacogenomics database: Genomics of Drug Sensitivity in Cancer (GDSC) (https://www.cancerrxgene.org/) [51]. In addition, Tumor Immune Dysfunction and Exclusion (TIDE) (http://tide.dfci.harvard.edu/) algorithm was implemented to predict immune checkpoint blockade therapy response between two groups [52].

\section{Gene set enrichment analysis (GSEA) in TCGA COAD/READ cohort.}

To explore the different KEGG pathways and hallmark gene sets between high- and low-CAF risk groups, GSEA was performed with The Molecular Signatures Database (MSigDB) (c2.cp.kegg.v7.3.symbols, h.all.v7.3.symbols) via fgsea $R$ package (version 1.12.0) [53, 54]. Pathways with an adjusted $\mathrm{P}<0.05$ were deemed to be significantly enriched.

\section{Nomogram construction and validation}

Univariate and successive multivariate Cox regression analyses were performed to ascertain whether CAF model was independent of several clinical characteristics via survival $R$ package. Subsequently, the nomogram was constructed based on the multivariate Cox regression coefficients of CAF signature and clinical variables in the TCGA training cohort. The concordance index (C-index) was calculated to validate the nomogram's predictive performance, and calibration curves were also plotted to examine the consistence between the predicted 1-, 3and 5-year OS probabilities and the actual observations (bootstrap-based 1000 iterations resampling validations).

\section{Human Protein Atlas (HPA) database and immunohistochemistry $(\mathrm{IHC})$ verification}

The protein expressions of these CAF signature genes in CRC tissues were analyzed in HPA online database (https://www.proteinatlas.org/), which aims to create a human proteome-wide map through integrated omics technologies [55].

For markers that are not available in HPA database, IHC will be further applied to examine the protein expression patterns in CRC samples who underwent radical rectal resection in Peking University First Hospital. The research was approved by ethics committee of Peking University First Hospital, and written informed consent was obtained from all subjects. Formalin-fixed paraffin-embedded surgical CRC specimens were collected and incubated with primary antibodies against CEBPD (sc-365546, Santa Cruz Biotechnology Inc., 1:200 dilution) and CXCL1 (ab89318, abcam, 1:200 dilution)) overnight at $4{ }^{\circ} \mathrm{C}$, followed by incubation with secondary antibody (PV-9000, Beijing ZSGB-BIO) at room temperature for $30 \mathrm{~min}$. Then, diaminobenzidine tetrachloride
(DAB) staining (10 min at room temperature; ZLI-9019; Beijing ZSGB-BIO) was applied for CEBPD and CXCL1 expressions visualizations.

\section{Real-time polymerase chain reaction (qPCR)}

Tissue microarrays containing 80 colon cancer samples (HColA095Su01) with the corresponding clinicopathological information were purchased from Shanghai Outdo Biotech (Shanghai, China) for prognostic verification. The mRNA expressions of the nine signature genes were quantified by qPCR using SYBR Green Premix (YEASEN, China) on the 7500 Sequence Detection System (Applied Biosystems, China). $\beta$-actin served as internal control for qPCR normalization, and the relative gene levels were calculated by $2^{-\Delta \Delta C T}$ method. The primer sequences of these genes were listed in Additional file 1: Table S1.

\section{Statistical analysis}

All statistical analyses and visualization were performed using R software v3.6.3 (https://www.r-project.org/). Wilcoxon test was applied for the comparisons between two groups. Survival analysis was performed using survival and survminer $\mathrm{R}$ packages. P-value less than 0.05 was regarded as statistical significance.

\section{Results}

\section{Single-cell RNA-seq profiling, clustering and markers identifications}

The overall study flow scheme was depicted in Fig. 1. After preprocessing scRNA-seq data based on the stringent quality control metrics as noted, 8696 high-quality cell samples isolated from the four discovery CRC tissues were screened and illustrated in Fig. 2a, and a strong positive correlation between numbers of detected genes (nFeature) and sequencing depth (total number of UMIs, nCount) was observed with the Pearson's correlation of 0.94 (Fig. 2b). We subsequently adopted t-SNE technique on the top 20 principal components to visualize the high dimensional scRNA-seq data, and successfully classified cells into fourteen subclasses, which were later annotated to acknowledged cell types using SingleR R package (Fig. 2c). The following major cell types were characterized: CD8 + T-cells, epithelial cells, macrophages, B-cells, fibroblasts, HSC and endothelial cells. In addition, the fibroblasts cluster was manually verified through wellacknowledged CAF markers (ACTA2, FAP, PDGFRB, CAV1, PDPN, PDGFRA, ZEB1, FOXF1, SPARC, MMP2, FN1) $[25,56]$, which further conformably confirmed the veracity of automated CAF cluster annotation (Fig. 6a, c). Furthermore, significant expressed marker genes across each group were identified with a threshold of $\operatorname{logFC}>1$ and $\operatorname{adjPval}<0.05$, and the top 10 significant differential 


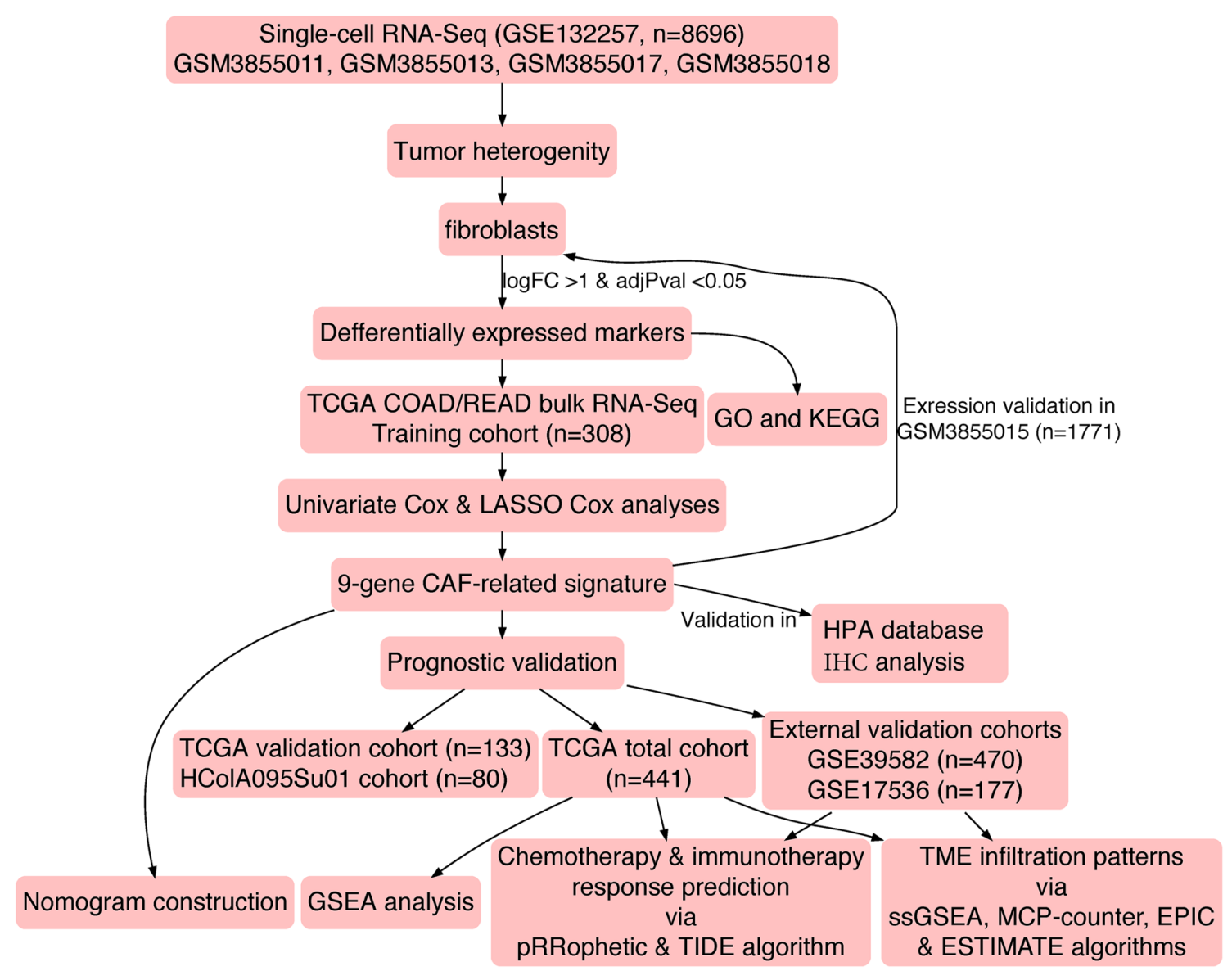

Fig. 1 The schematic diagram of this study

markers of each cluster was displayed via heatmap (Fig. 2d).

Meanwhile, similar analyses were performed on the validation sample (GSM3855015), a total of 1771 highquality cell samples were screened (Additional file 2: Fig. S1a), the Pearson's correlation between nFeature and nCount was 0.95 (Additional file 2: Fig. S1b). Eleven clusters were classified via t-SNE technique and annotated as six types of cells (CD8 + T-cells, epithelial cells, macrophages, B-cells, fibroblasts and HSC; Additional file 2: Fig. S1c), and the top 10 significant differential markers of each cluster was visualized in Supplementary Fig. 1d. Similarly, the fibroblasts cluster was manually authenticated through the above summarized CAF markers (Additional file 3: Fig. S2a, c).

\section{GO and KEGG functional downstream analyses of fibroblast marker genes}

We conducted GO and KEGG enrichment analyses to investigate the correlative functions and pathways of the above 208 genes in cluster 10 (fibroblast cluster).
As shown in Fig. 3a, extracellular matrix organization, extracellular structure organization, collagen-containing extracellular matrix and extracellular matrix structural constituent were the main significantly enriched GO terms. Figure $3 \mathrm{~b}$ exhibited the top 30 enriched KEGG pathways, which involved mainly in the desmoplastic and immune processes, such as focal adhesion, cell adhesion molecules, proteoglycans in cancer, Th1 and Th2 cell differentiation, antigen processing and presentation and leukocyte transendothelial migration pathways. These enrichment terms strengthened that the cluster was appropriately annotated and reliable for marker screening.

\section{Nine-gene prognostic CAF signature construction and verification}

In the TCGA training cohort, by inputting the 208 differential CAF marker genes identified above into univariate Cox regression analysis, a total of 41 genes were exhibited with $\mathrm{P}<0.1$. LASSO Cox regression algorithm was then performed on these genes, the lambda. 


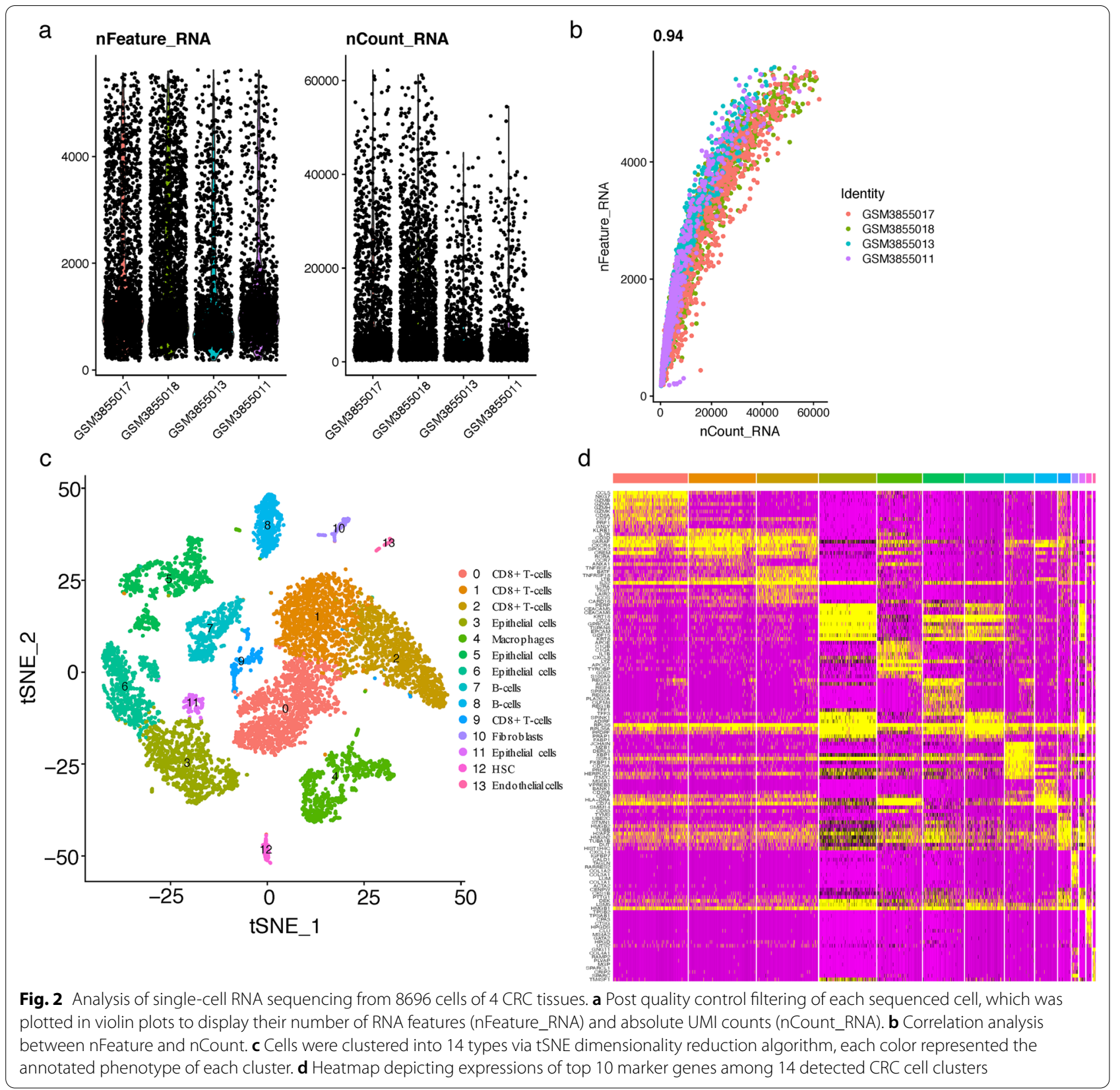

min was determined as the optimal lambda value by tenfold cross-validations, and 9 prognostic genes with non-zero coefficients were successfully identified (Fig. 4a, b). A nine-gene CAF signature was subsequently constructed based on each gene's expression level and its coefficient: risk score $=(0.204341111$ * expression of CEBPD $)+(0.054785445$ * expression of CSRP2 $)+(-0.123917745$ * expression of CXCL1 $)+(0.005667789 *$ expression of HSPB1 $)+(0.044691324 *$ expression of PPP1R14A $)+(0.014977193$ " expression of S100A13) $+(-0.137566435 *$ expression of SPINK 1$)+(0.230258936 *$ expression of TIMP1 $)+(0.058696341$ * expression of TIMP2). Among the 9 prognostic genes, seven genes (HSPB1, S100A13, PPP1R14A, CSRP2, TPM2, CEBPD and TIMP1) were regarded as risk-related genes $(H R>1)$, while SPINK1 and CXCL1 were considered as protective genes $(\mathrm{HR}<1)$ (Fig. 4c). Based on this risk formula, CAF risk score of each patient was calculated, and heatmaps illustrating the risk score and expression levels of the 9 genes in each cohort were displayed in Fig. $4 \mathrm{~d}-\mathrm{i}$. Patients in 

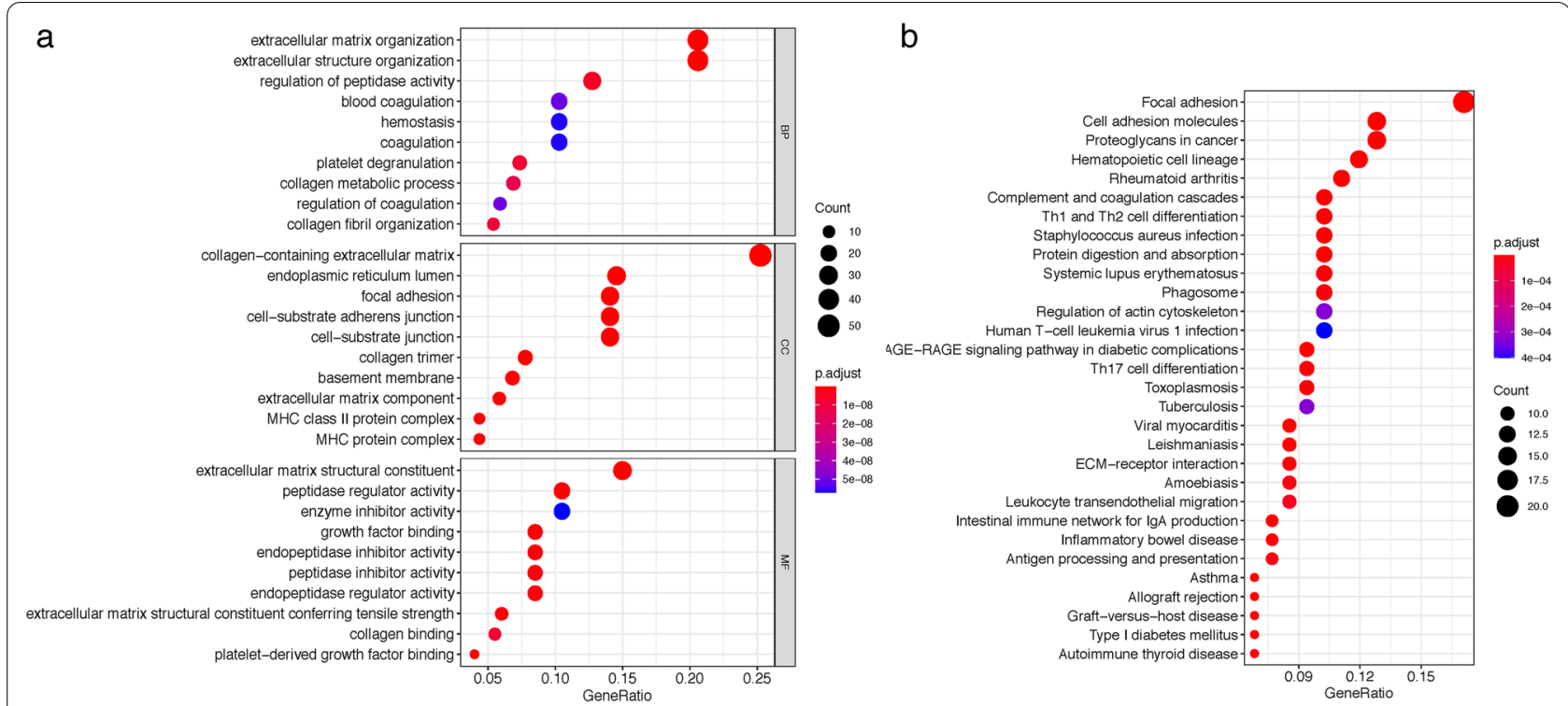

Fig. 3 Bubble map of a the top $10 \mathrm{GO}$ terms and $\mathbf{b}$ KEGG pathway enrichment analysis of 208 significant expressed marker genes in the fibroblasts cluster

TCGA training, TCGA total, GSE39582 and GSE17536 cohorts were divided into low- and high-CAF risk groups in the light of their median risk scores. The pairwise comparison of OS in different risk groups was investigated by log-rank test. Kaplan-Meier curves revealed that high CAF risk group had significantly unfavorable survival outcomes compared with the low CAF risk group (TCGA training cohort, hazard ratio $(\mathrm{HR})=4.178$, 95\% CI: 2.222-7.854, log-rank $\mathrm{P}<0.001$, Fig. 5a; TCGA total cohort, $\mathrm{HR}=3.272,95 \% \mathrm{CI}: 2.008-5.332$, log-rank $\mathrm{P}<0.001$, Fig. 5c; GSE39582 cohort, $\mathrm{HR}=1.496$, 95\% CI: $1.078-2.075$, log-rank $\mathrm{P}=0.016$, Fig. 5d; GSE17536 cohort, $\mathrm{HR}=1.924,95 \%$ CI: 1.197-3.092, log-rank $\mathrm{P}=0.007$, Fig. 5e). In TCGA validation and $\mathrm{HCo}-$ lA095Su01 datasets, the optimal cutoff was determined by "sur_cutpoint" function of survminer $\mathrm{R}$ package, and higher CAF risk group patients also revealed worse OS than lower CAF risk group in TCGA validation $(\mathrm{HR}=2.567,95 \% \mathrm{CI}: 1.237-5.326$, log-rank $\mathrm{P}=0.011$, Fig. $5 \mathrm{~b})$ and HColA095Su01 (HR $=2.187,95 \% \mathrm{CI}$ : $1.08-$ 4.433, log-rank $\mathrm{P}=0.03$, Fig. $5 \mathrm{f}$ ) cohorts. In summary, this CAF model could serve as a reliable prognostic predictor for CRC patients.

Subsequently, expression characteristics of the nine markers among clusters in single-cell RNA sequencing profile were visualized via violin and bubble plots, CSRP2, PPP1R14A and TPM2 were up-expressed mainly in fibroblasts, while SPINK1 was down-regulated and expressed exclusively in epithelial cells. The other six markers were upregulated in fibroblasts cluster and revealed multiple expression forms among clusters (Fig. 6b, c). Moreover, similar results were reached through the external expression validation in GSM3855015, which confirmed the robustness of these genes as markers of CAFs (Additional file 3: Fig. S2b, c).

\section{TME infiltration patterns with $C A F$ risk group}

By running ssGSEA, MCP-counter, EPIC and ESTIMATE algorithms, we investigated the correlation of CAF risk score and TME constituents at bulk RNAsequencing level. As shown in Fig. 7, pooled results of heatmaps and Wilcoxon analyses on TCGA COAD/ READ, GSE39582 and GSE17536 datasets revealed the stromal and immune scores, as well as the infiltrations of several TME contents like fibroblasts, Th1 cells, Tgd, Tem, NK cells, neutrophils, Mast cells, Macrophages, iDC, cytotoxic cells and CD8 T cells were higher in high CAF risk group.

\section{CAF-related signature was predictive to chemotherapy and immunotherapy response}

We next investigated the practicability of the model in guiding systemic therapies. Firstly, estimated IC50 values were calculated by pRRophetic algorithm to predict the different chemotherapy responses of CAF-associated high and low risk groups. Based on the GDSC cancer cell line database, we found that higher CAF risk score increased the sensitivity of 6 types of anticancer drugs (camptothecin, docetaxel, gefitinib, gemcitabine, pazopanib, sunitinib) in TCGA COAD/READ, GSE39582 and GSE17536 datasets (Wilcoxon test, all $\mathrm{P}<0.01$; Fig. 8ac). Subsequently, using the TIDE online algorithm, we 


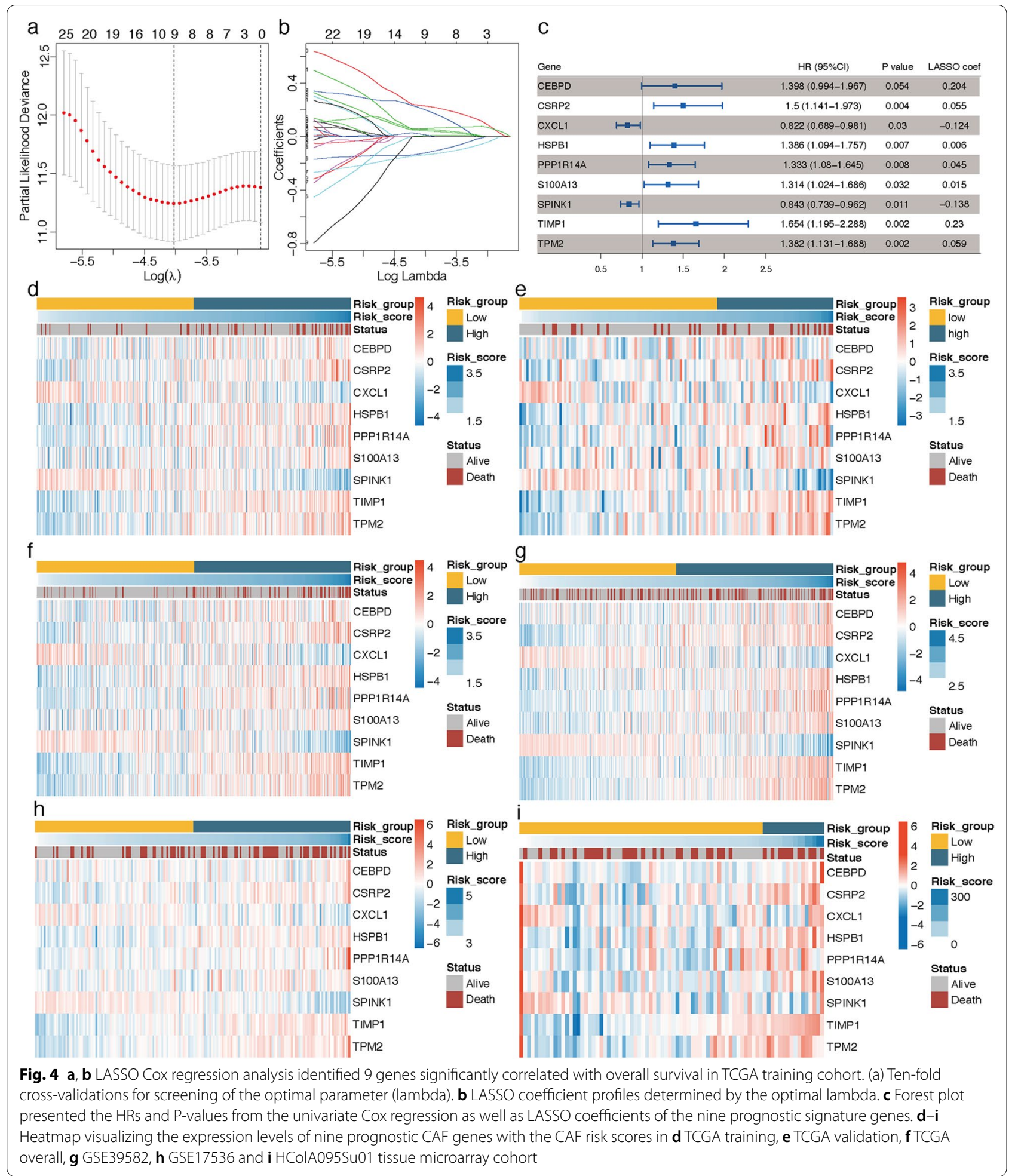

predicted the probability of response to immune checkpoint inhibitors in the three datasets. In the TCGA cohort, as shown in Fig. 8D, low-CAF risk patients $(54.3 \%, 120 / 221)$ were more reactive to immunotherapy compared with high-CAF risk patients $(26.36 \%, 58 / 220)$ (Chi-Square test, $\mathrm{P}<0.001$ ), in addition, responders presented markedly lower CAF scores compared to nonresponders (Wilcoxon test, $\mathrm{P}<0.001$ ). Similarly, in the 

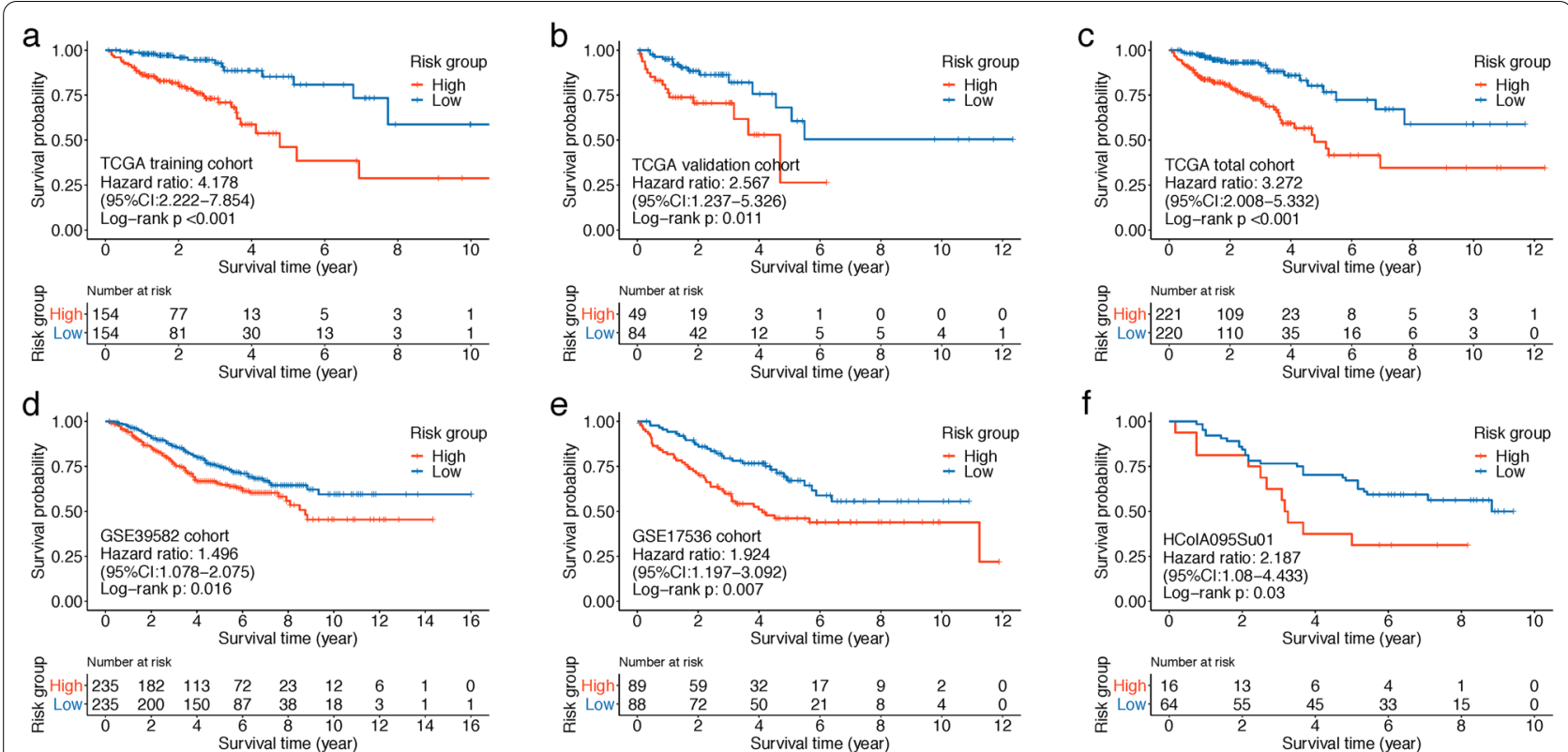

Fig. 5 Kaplan-Meier curves displayed that high-CAF risk group had worse overall survival (OS) than low-CAF risk group in a TCGA COAD/READ training, b TCGA validation, c TCGA overall, d GSE39582, e GSE17536 and f HColA095Su01 tissue microarray cohorts

GSE39582 (Fig. 8e) and GSE17536 (Fig. 8f) validation cohorts, low-CAF risk group patients $(68.51 \%, 161 / 235$ in GSE39582; 64.05\%, 57/89 in GSE17536) were also more responsive to immunotherapy than high-risk patients $(28.51 \%, 67 / 235$ in GSE39582; 26.14\%, 23/88 in GSE17536) (Chi-Square test, both $\mathrm{P}<0.001$ ), and the responders presented significantly lower CAF scores in the two cohorts (Wilcoxon test, both $\mathrm{P}<0.001$ ). These results implied that while high CAF score was correlated with increased chemotherapy sensitivity, immunotherapy might be more effective in low CAF score CRC patients.

\section{Functional assessment of CAF signature}

Since the designed CAF signature was highly correlated with adverse prognosis and refractory immunotherapy response, we then investigated the functional pathways in this model through GSEA analysis. Patients in the TCGA COAD/READ cohort were separated into high- and low-risk groups based on their median CAF risk score as the cutoff value. We found several immunerelated KEGG gene sets were enriched in high CAF risk group, including antigen processing and presentation, $\mathrm{B}$ cell receptor signaling pathway, $\mathrm{T}$ cell receptor signaling pathway, chemokine signaling pathway, leukocyte transendothelial migration, primary immunodeficiency, calcium signaling pathway, cell adhesion molecules cams, cytokine-cytokine receptor interaction (Fig. 9a). In addition, several hallmark gene sets, including angiogenesis, complement, epithelial mesenchymal transition, hypoxia, inflammatory response and myogenesis were also significantly enriched in high CAF risk group (Fig. 9b).

\section{CAF-related signature was an independent prognostic factor in CRC patients.}

Univariate and multivariate Cox regression analyses were sequentially carried out in each cohort to examine whether the prognostic value of CAF-related gene signature was independent of other features including age and TNM stage. As shown in Table 1, in TCGA training, TCGA total, GSE39582, GSE17536 and HCo1A095Su01 cohorts, both univariate and multivariate Cox analysis manifested the CAF signature were independent prognostic factors. However, after adjusting for age and TNM stage parameters, the prognostic

(See figure on next page.)

Fig. 6 a Recognized and $\mathbf{b}$ the nine identified CAF markers expressions in CRC single-cell clusters. c Bubble plot visualizing genes expression characteristics in single-cell RNA sequencing profile. Cell phenotypes were listed on y-axis, recognized CAF markers (left part of the dotted line) as well as the identified nine prognostic markers (right part of the dotted line) were listed along the x-axis. Dot size reflects each gene's expressing percentage of each cluster's cells; dot color represents the expression level 


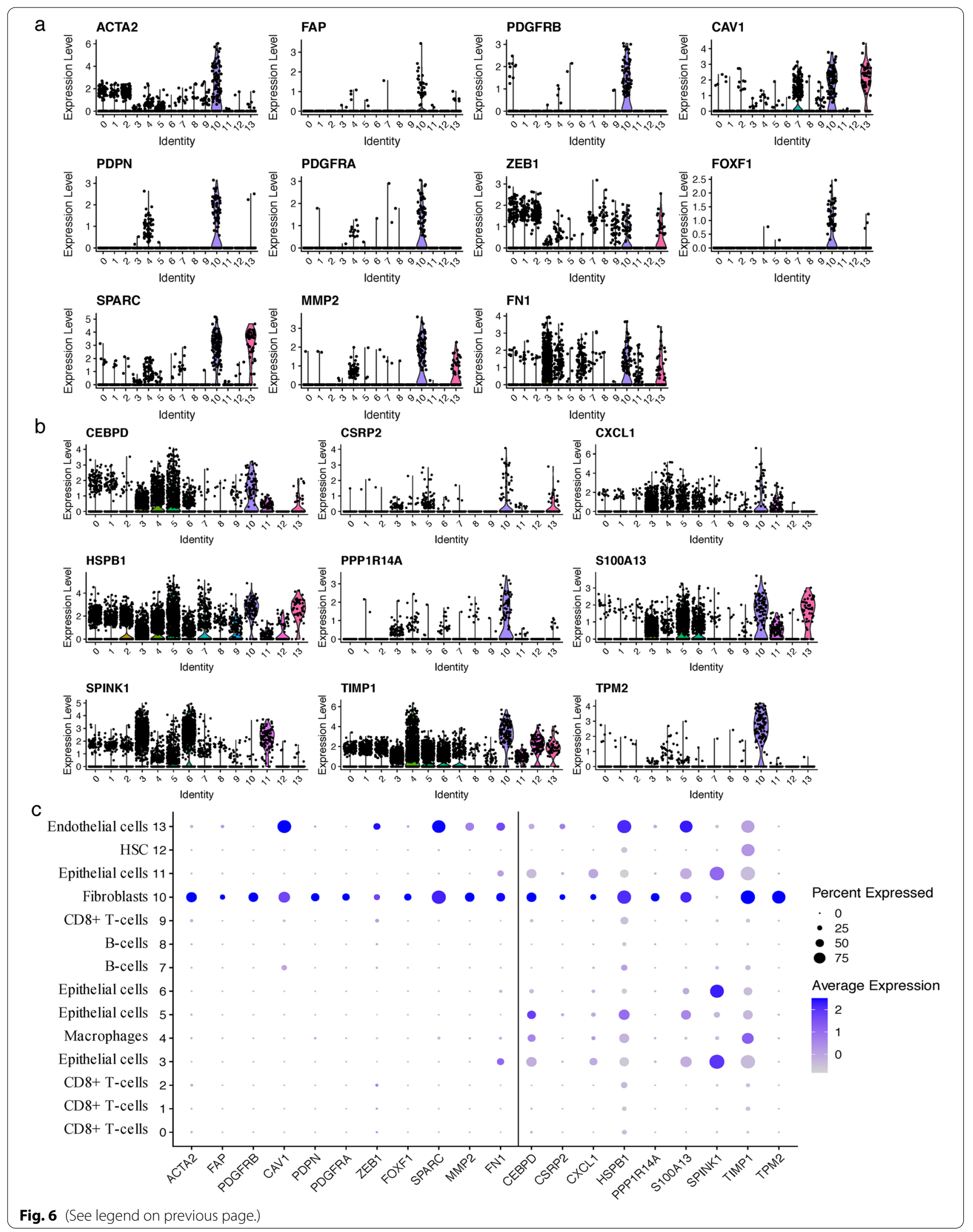




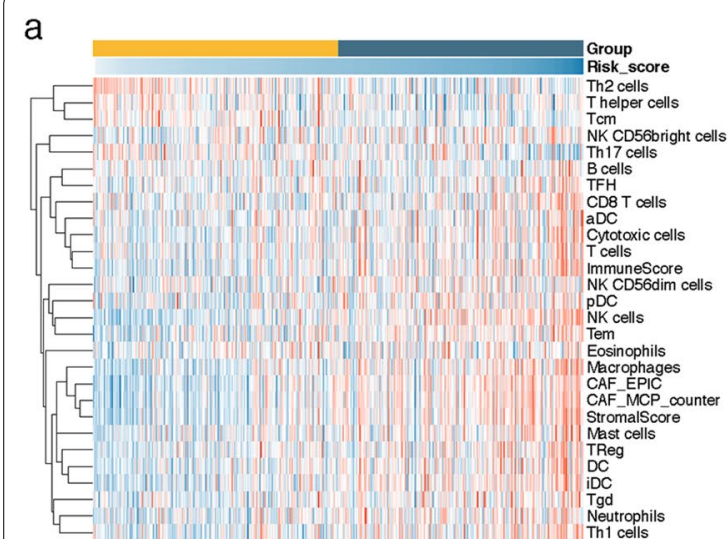

b

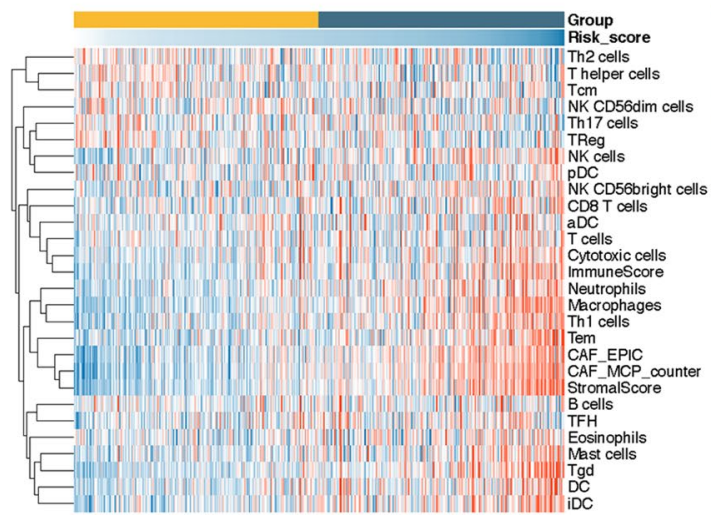

C

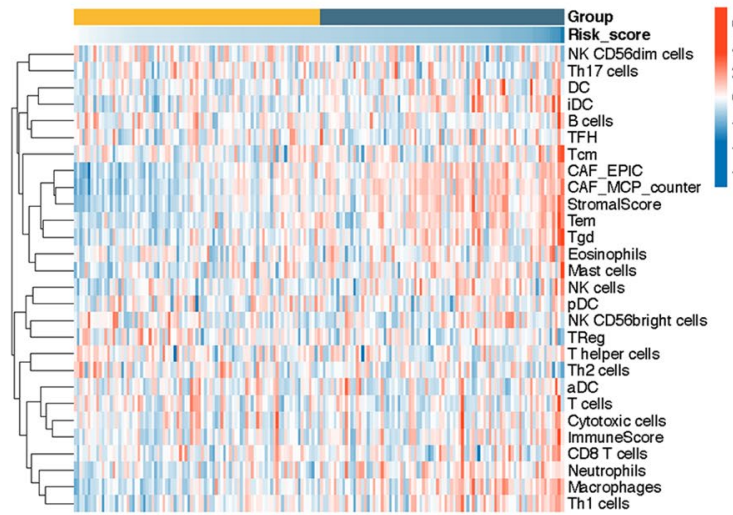

d

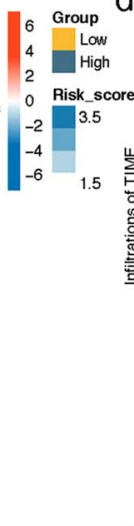

e
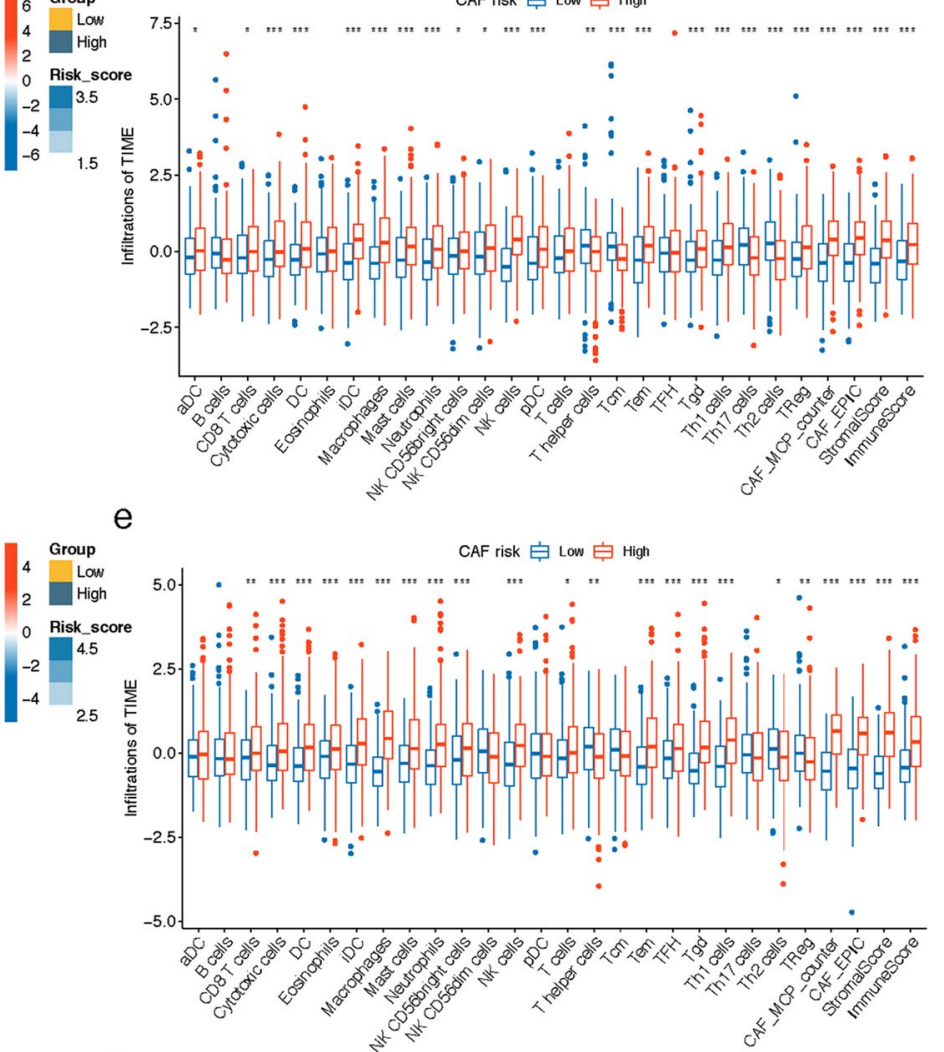

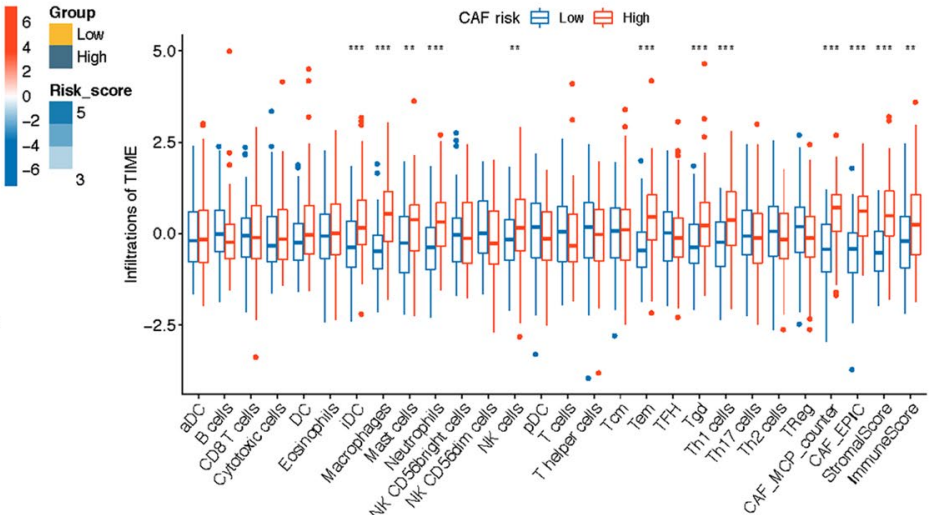

Fig. 7 a-c Heatmap illustrating the distributions of 24 immune cell subsets, fibroblasts, stromal and immune scores assessed via sSGSEA, MCP-counter, EPIC and ESTIMATE algorithms in a TCGA COAD/READ, b GSE39582 and c GSE17536 cohort. (d-f) Wilcoxon analysis of the differing TME subtype distributions (z-score standardized) between high- and low-CAF risk groups in d TCGA COAD/READ, e GSE39582 and f GSE17536 cohort $\left.{ }^{*} P<0.05,{ }^{* *} P<0.01,{ }^{* * *} P<0.001\right)$

(See figure on next page.)

Fig. 8 a-c The IC50 values of six anti-cancer drugs were predicted by pRRophetic algorithm and were Z-score normalized. The difference between high- and low-CAF risk groups of each drug in a TCGA COAD/READ, b GSE39582 and c GSE17536 cohort was compared by Wilcoxon test (** $\mathrm{P}<0.01$, $\left.{ }^{* * *} \mathrm{P}<0.001\right)$. $\mathbf{d}-\mathbf{f}$ TIDE analysis for predicting the likelihood of clinical response to immune checkpoint inhibitors in $\mathbf{d} T C G A C O A D / R E A D, \mathbf{e}$ GSE39582 and $\mathbf{f}$ GSE17536 cohort. Patients with a lower CAF risk score are more likely to respond from immune therapy 


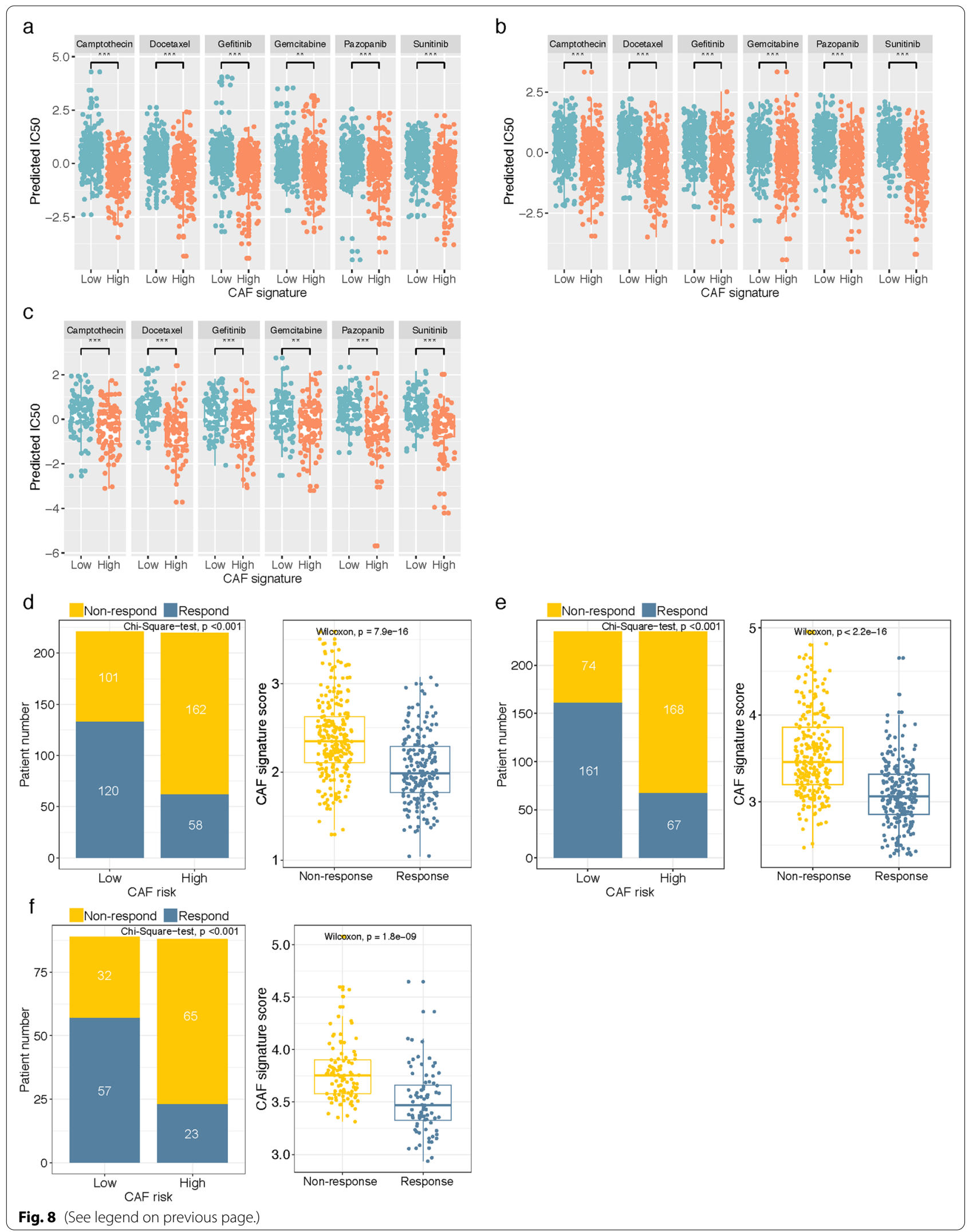




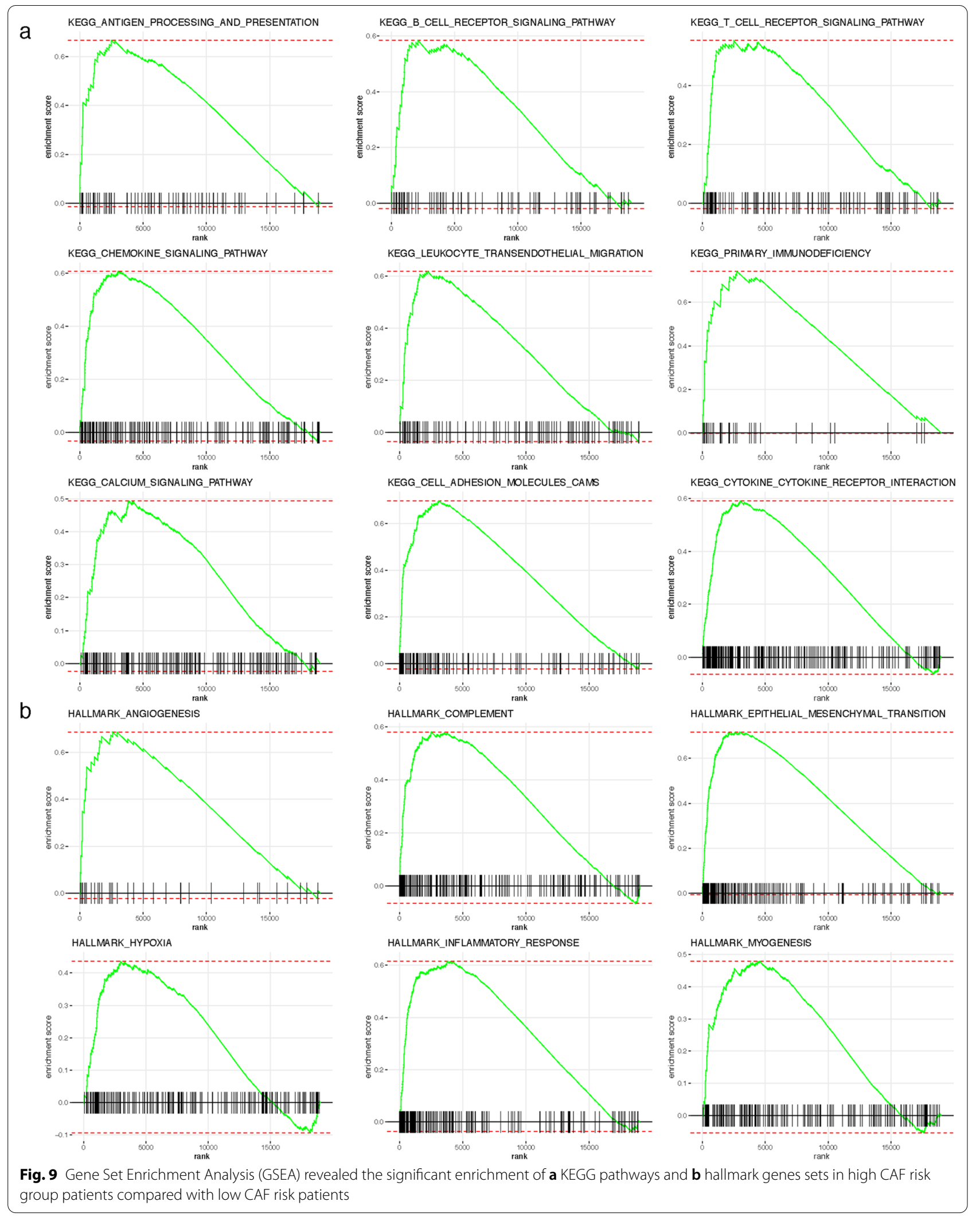


Table 1 Univariate and multivariate Cox proportional hazards regression analysis on OS

\begin{tabular}{|c|c|c|c|c|c|c|}
\hline & \multicolumn{3}{|c|}{ Univariate analysis } & \multicolumn{3}{|c|}{ Multivariate analysis } \\
\hline & HR & $95 \% \mathrm{Cl}$ & $\mathbf{P}$ & HR & $95 \% \mathrm{Cl}$ & $\mathbf{P}$ \\
\hline \multicolumn{7}{|c|}{ TCGA training cohort } \\
\hline Age & 1.023 & 0.999-1.049 & 0.06 & 1.033 & $1.008-1.058$ & 0.009 \\
\hline TNM stage & 2.67 & $1.918-3.718$ & $<0.001$ & 2.73 & $1.911-3.9$ & $<0.001$ \\
\hline CAF signature & 4.418 & $2.521-7.744$ & $<0.001$ & 3.161 & $1.828-5.468$ & $<0.001$ \\
\hline \multicolumn{7}{|c|}{ TCGA validation cohort } \\
\hline Age & 1.061 & $1.024-1.099$ & 0.001 & 1.058 & $1.023-1.094$ & 0.001 \\
\hline TNM stage & 2.363 & $1.566-3.564$ & $<0.001$ & 2.391 & $1.566-3.651$ & $<0.001$ \\
\hline CAF signature & 2.323 & $1.094-4.932$ & 0.028 & 1.334 & $0.609-2.920$ & 0.471 \\
\hline \multicolumn{7}{|l|}{ TCGA total cohort } \\
\hline Age & 1.036 & $1.016-1.057$ & $<0.001$ & 1.039 & $1.02-1.059$ & $<0.001$ \\
\hline TNM stage & 2.553 & $1.973-3.304$ & $<0.001$ & 2.568 & $1.962-3.361$ & $<0.001$ \\
\hline CAF signature & 3.45 & $2.212-5.381$ & $<0.001$ & 2.261 & $1.454-3.516$ & $<0.001$ \\
\hline \multicolumn{7}{|l|}{ GSE39582 cohort } \\
\hline Age & 1.039 & $1.024-1.053$ & $<0.001$ & 1.037 & $1.023-1.052$ & $<0.001$ \\
\hline TNM stage & 1.688 & $1.315-2.167$ & $<0.001$ & 1.646 & $1.272-2.13$ & $<0.001$ \\
\hline CAF signature & 1.744 & $1.277-2.382$ & $<0.001$ & 1.455 & $1.055-2.007$ & 0.022 \\
\hline \multicolumn{7}{|l|}{ GSE17536 cohort } \\
\hline Age & 1.006 & $0.988-1.025$ & 0.492 & 1.027 & $1.006-1.047$ & 0.01 \\
\hline TNM stage & 2.855 & $2.112-3.859$ & $<0.001$ & 3.323 & $2.373-4.653$ & $<0.001$ \\
\hline CAF signature & 2.553 & $1.413-4.613$ & 0.002 & 3.018 & $1.644-5.539$ & $<0.001$ \\
\hline \multicolumn{7}{|c|}{ HColA095Su01 cohort } \\
\hline Age & 0.995 & $0.969-1.022$ & 0.704 & 0.997 & $0.97-1.026$ & 0.86 \\
\hline TNM stage & 1.069 & $0.631-1.811$ & 0.803 & 1.239 & $0.714-2.148$ & 0.446 \\
\hline CAF signature & 1.003 & $1.001-1.005$ & 0.011 & 1.003 & $1.001-1.005$ & 0.014 \\
\hline
\end{tabular}

OS overall survival, HR hazard ratio, CI Confidence interval, CAF Cancer associated fibroblast

value of CAF signature was limited in TCGA validation cohort $(\mathrm{HR}=1.334,95 \% \mathrm{CI}: 0.609-2.920, \mathrm{P}=0.471)$, we considered this was due in part to the relative small sample size in this cohort.

Based on the multivariate Cox regression coefficients of nine-CAF-related gene signature and clinical traits (age and TNM stage) in the TCGA training cohort, we built a prognostic nomogram for clinicians to quantitatively predict 1,3 and 5 -year OS probabilities of CRC patients (Fig. 10a). The C-index of the nomogram was $0.813(95 \% \mathrm{CI}=0.746-0.88)$ in TCGA training set, $0.772(95 \% \mathrm{CI}=0.677-0.867)$ in TCGA validation set, $0.792(95 \% \mathrm{CI}=0.736-0.848)$ in TCGA total set, 0.658 $(95 \% \mathrm{CI}=0.611-0.705)$ in GSE39582 set, and $0.78(95 \%$ $\mathrm{CI}=0.726-0.834)$ in GSE17536 set. The calibration curves manifested the model's predictions of 1-, 3- and 5 -year OS probabilities were favorably consistent with the ideal predictions (gray line) in all datasets (Fig. 10b). These results demonstrated the aggregated nomogram model could serve as a reliable tool for OS predictions of CRC patients.
Study of the expression patterns of CAF-related signature genes at protein levels via HPA database and IHC analyses Finally, with respect to CRC tissue protein expression levels, the immunohistochemical results from HPA database indicated that protein expression of CSRP2, HSPB1, PPP1R14A, S100A13, TIMP1 and TPM2 was higher in CRC stroma (Fig. 11a-f), while SPINK1 was weakly expressed in interstitial areas (Fig. 11g), and there was no immunohistochemical data for the other 2 genes (CEBPD and CXCL1) in HPA database. Hence, IHC analyses were performed on these two genes, and the examples of IHC staining of CEBPD and CXCL1 were shown in Fig. 11h, i. The expressions of CEBPD (Fig. 11h) and CXCL1 (Fig. 11i) were also found mainly in stromal spaces of CRC tissues.

\section{Discussion}

The recognized tumor-promoting capacity of CAFs makes them a prospective immunotherapy target $[9,11$, 57]. However, the clinical applications remain challenging owing to the lack of effective targetable biomarker, which motivated us to investigate the novel CAF markers 


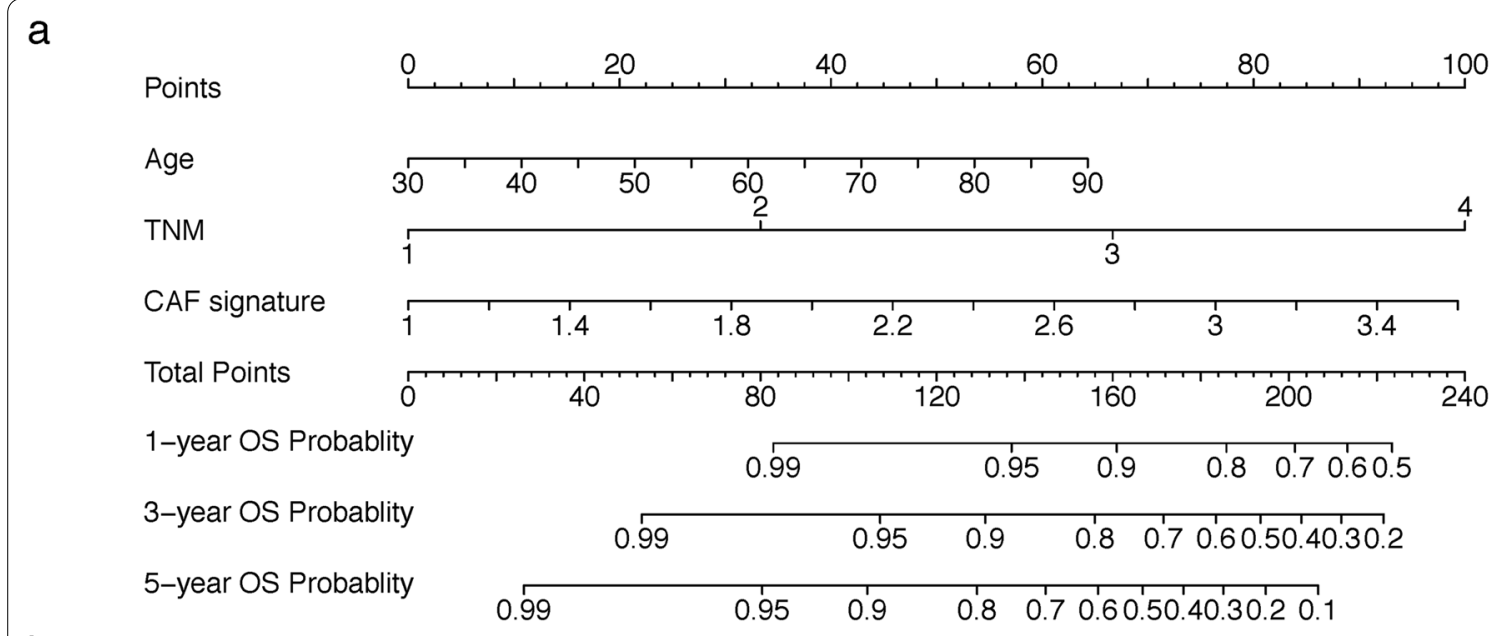

b
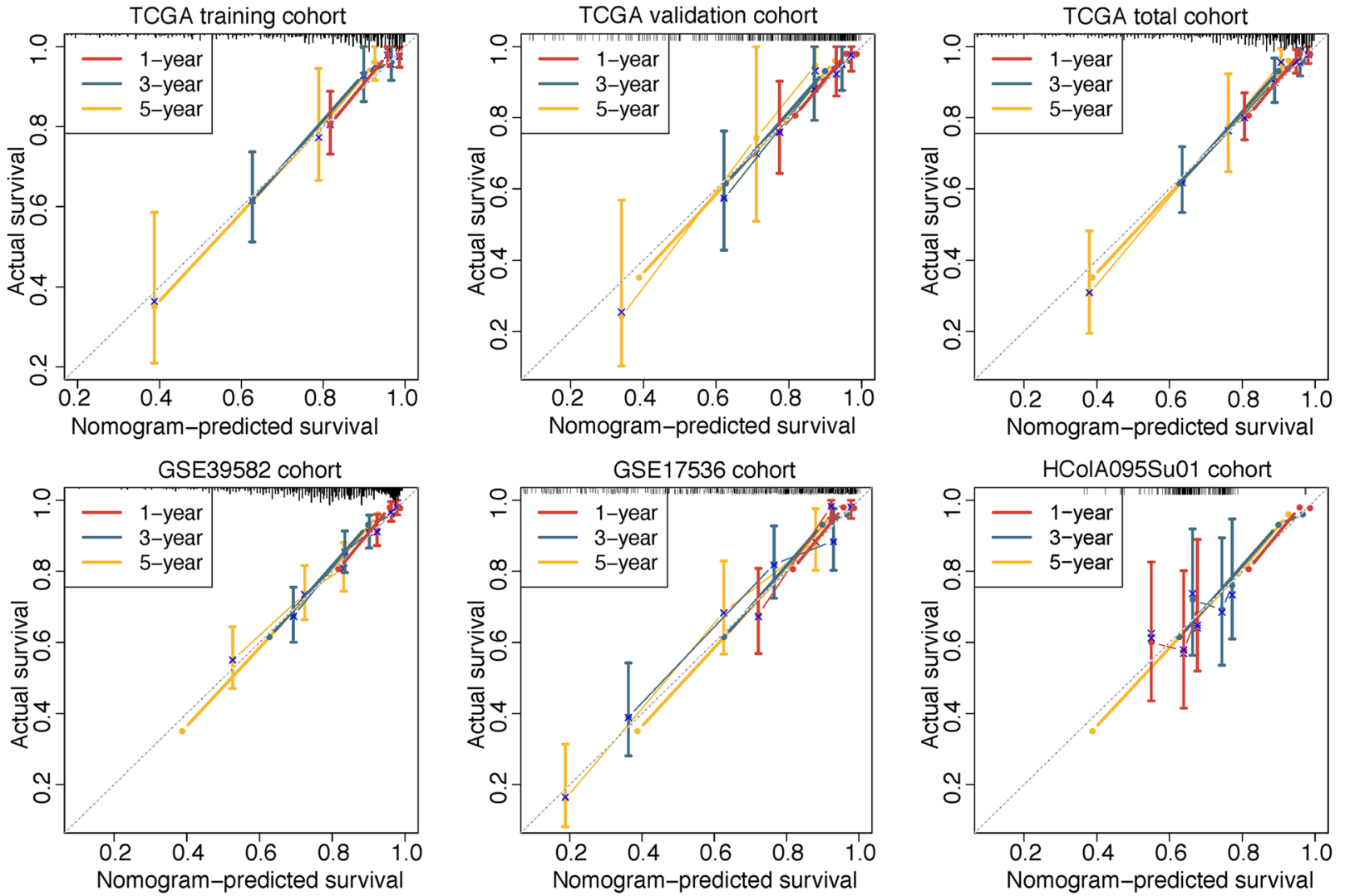

Fig. 10 Nomogram construction and calibration plot validations. a Nomogram based on age, TNM stage and CAF signature for 1-, 3- and 5-year OS predictions. b Calibration curves for testing the agreement between 1-,3- and 5-year predicted overall survival and actual observations in TCGA training, TCGA validation, TCGA entire, GSE39582, GSE17536 and HColA095Su01 cohorts

in CRC. In this study, by analyzing single-cell genome of the GEO CRC patient datasets, we clearly revealed the fibroblasts subset and characterized 208 fibroblast markers with highly altered expressions that could not be discriminated in bulk RNA-seq. Since CAFs revealed a high level of heterogeneity [58], a model composed of multiple biomarkers would achieve an improved prognostic efficacy over the individual biomarker. Therefore, combined with the integrated analysis of TCGA COAD/ READ, GSE39582 and GSE17536 bulk sequencing projects as well as HColA095Su01 experimental verification cohort, we for the first time established and validated a 


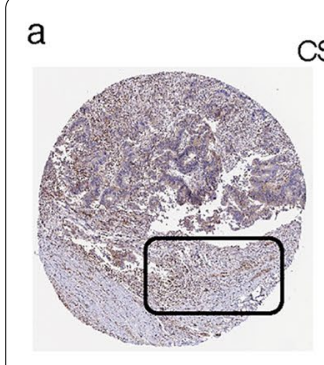

C

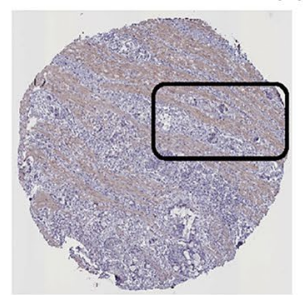

e

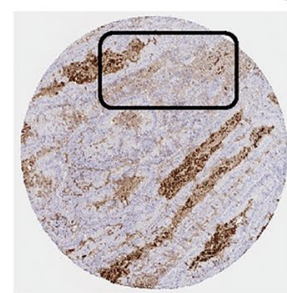

g

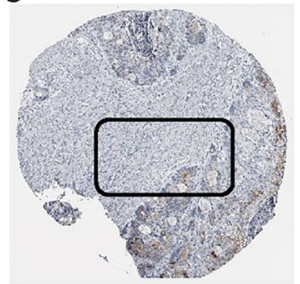

PPP1R14A

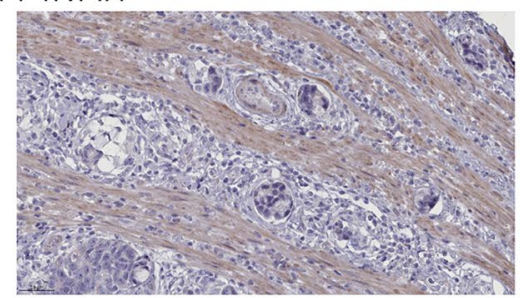

TIMP1

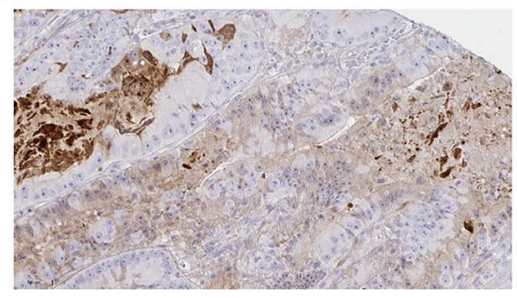

SPINK1

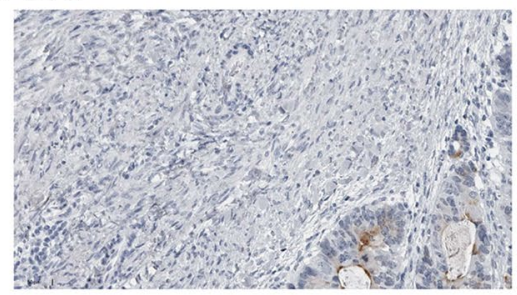

CXCL1 b

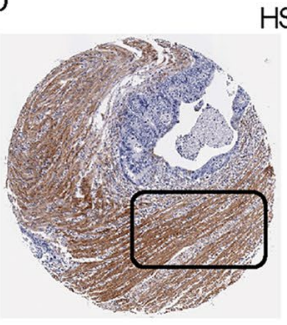

d

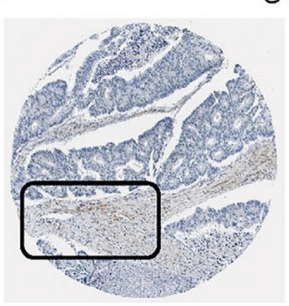

f

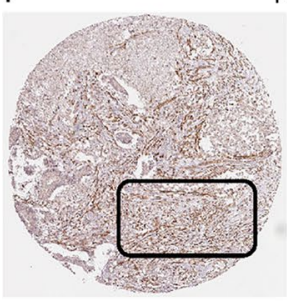

h

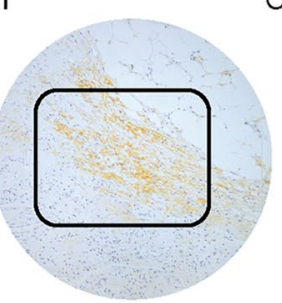

HSPB1

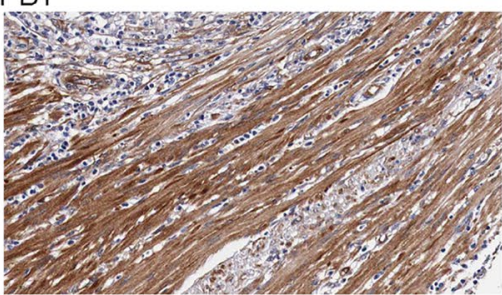

S100A13

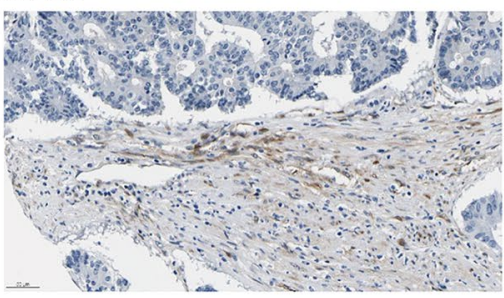

TPM2

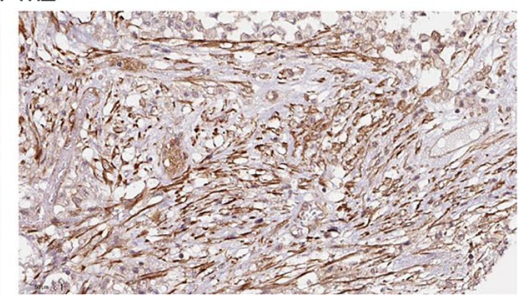

CEBPD
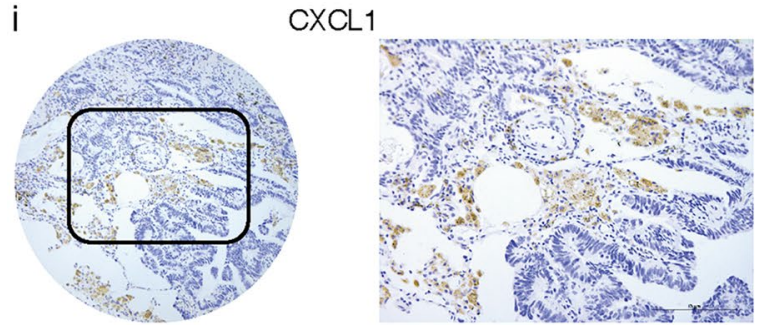

Fig. 11 (a-g) Immunohistochemistry showing the protein expressions of a CSRP2, b HSPB1, c PPP1R14A, d S100A13, e TIMP1, f TPM2 and $\mathbf{g}$ SPINK1 based on the Human Protein Atlas (HPA) database. $\mathbf{h}, \mathbf{i}$ Immunohistochemistry images of $\mathbf{h}$ CEBPD and $\mathbf{i}$ CXCL1 protein expressions in CRC tissues

robust nine-gene CAF-related molecular signature capable of predicting prognosis, estimating TME stromal and fibroblasts components and therapy response for CRC patients. Univariate and multivariate Cox regression analyses verified the CAF-related signature was an independent risk factor associated with OS. To improve the predictive efficacy of the signature and facilitate clinical application, we subsequently constructed and validated a nomogram based on age, TNM stage and CAF signature for clinical practicality to predict OS.

In this study, we observed the immune and stromal cells infiltrations were more abundant in the high CAF risk group, especially several immunosuppressive types like fibroblasts, macrophages and mast cells. The 
effectiveness of most immunotherapies depends on the abundant infiltrations of CD8 $+\mathrm{T}$ cells in the tumors [59]. However, while high CAF risk group harbored relative richer infiltrations of Th1 cells, cytotoxic cells and CD8 T cells, TIDE algorithm manifested that high CAF risk CRC patients were more likely not to respond to anti-PD-1 and anti-CTLA-4 therapies in all three datasets. This could be partly explained by the findings from Ford et al. that CAFs modulate immunotherapies resistance specifically by discharging $\mathrm{CD} 8+\mathrm{T}$ cells from tumor mass to tumor margin [60], meanwhile, the remodeling ECM constructed interactively by CAFs and cancer cells serve as the physical hamper against the penetration of tumoricidal immune cells as well as the delivery of anticancer agents to solid tumors [20,61]. Interestingly, pRRophetic algorithm indicated that high-CAF risk CRC patients were more sensitive to several conventional chemotherapy agents than low-CAF risk patients. Researchers have demonstrated the anti-tumor immune efficacy of traditional chemotherapeutics [62]. For example, McDonnell et al. reported the standard-dose gemcitabine would enhance the tumor-associated antigens cross-presentation efficacy of tumor-resident dendritic cells to enable the reactivation capacity of tumor-infiltrating $\mathrm{CD} 8+\mathrm{T}$ cells [63]. We proposed a promising therapeutic strategy of combining conventional chemotherapy, natural-based substitutes [64, 65] and CAF-targeting immunotherapy to stimulate intratumoral CD8 + T-cell penetrations and resensitize high CAF-risk tumors to the current $\mathrm{T}$ cell based immunotherapies. However, further studies are needed for the design of synergistic therapies.

For the CAF-related genes in this well-established signature, scRNA-seq displayed TIMP1 expressed mostly in fibroblasts, epithelial cells, macrophages and endothelial cells. Many studies have identified dysregulation of TIMP1 expressions contributed critically to pro-tumor inflammation initiation, matrix remodeling and fibrosis development [66-68]. Illemann et al. reported that TIMP1 was generally expressed in $\alpha$-SMA-positive myofibroblasts in both primary CRC and liver metastases, and promotes the anti-apoptosis and pro-angiogenesis activities [69]. TIMP1 has also been verified to promote intra-tumoral CAFs infiltration, proliferation and migration by activating ERK $1 / 2$ kinase in CAFs [70]. In addition, upregulated TIMP1 in the cancerous CAF stroma would participate the vascular remodeling process and enhance the invasions of colorectal cancer cells [71]. Moreover, knockdown of TIMP1 could promote apoptosis of colon cancer cells via BCL2-Associated Agonist Of Cell Death (BAD) mediated phosphoration pathway and suppress the migration and invasion of cancer cells through downregulating Fibronectin and upregulating E-cadherin
[72]. As the only downregulated gene in fibroblasts cluster, SPINK1 expressed mainly in epithelial cells and was regarded as a protective marker, which was consistent with previous studies [73-75]. However, in vitro experiments exhibited that SPINK1 contributed significantly to proliferation and invasion of CRC cell lines $[76,77]$. This discrepancy could be partly explained by the concurrent expressions of SPINK1 and EGFR, which exerts distinct functions in CRC tissue [74]. The inconsistency also occurred in CXCL1, except for one study that demonstrated CXCL1 was a protective gene [78], other studies demonstrated that high CXCL1 expression in CRC epithelium correlated with adverse clinicalpathological characteristics and poor prognosis $[79,80]$. CXCL1 is an inflammatory chemokine secreted mainly by CRC epithelia and myofibroblasts and is capable of driving tumor initiation and progression [81]. More experiments are needed to further validate the prognostic value of CXCL1 in CRC patients. Our results displayed PPP1R14A, TPM2 and CSRP2 were mainly expressed in fibroblasts cluster. PPP1R14A mRNA codes protein CPI-17, which directly deactivates tumor suppressor merlin (encoded by neurofibromatosis type 2 gene NF2) through merlin phosphorylation and tumorigenic Ras signaling activation $[82,83]$, and PPP1R14A has been reported to be aberrantly methylated in CRC [84] and serves as an epigenetic biomarker for CRC early detection [85]. A recent single-cell sequencing analysis from Zhou et al. also identified TPM2 as fibroblast-specific marker associated unfavorable prognosis in CRC [86]. Furthermore, Wang et al. reported the knockdown of CSRP2 would lead to the transformation from fibroblasts into CAFs and enhance their proliferation and migration capacities in gastric cancer [87], while its function in CRC fibroblasts still remains unclear. We showed CEBPD expressed mainly in epithelial cells, macrophages and fibroblasts. Chi et al. reported that CEBPD activation in M2 macrophages and myofibroblasts/CAFs led to the acquisition of chemoresistance and significantly promoted sphere-forming ability, stemness, invasion and metastasis in both responsive and drug-resistant breast cancers [88]. Wang et al. found CEBPD participated the integration of EMT and lipid metabolism signaling to promote lung adenocarcinoma metastasis [89]. S100A13 is a calcium binding protein gene [90], the digenic mutations of S100A13 would break calcium homeostasis, distort ECM and result in progression of lung fibrosis [91]. In addition, S100A13 is regarded as an angiogenic and prognostic biomarker in melanoma [92] and astrocytic gliomas [93]. Lee et al. demonstrated that HSPB1 is secreted from endothelial cells and physiologically modulates the balance of angiogenesis 
through interacting with vascular endothelial growth factor (VEGF). During the tumor-induced angiogenesis process, however, VEGF is overwhelmingly increased, yet the concomitant increase of HSPB1 is incapable of balancing the pathological angiogenesis, therefore contributes to tumor progression [94]. Nevertheless, the effects of CEBPD, S100A13 and HSPB1 on fibroblastinduced tumorigenesis in CRC have never been elucidated, which necessitate further investigations.

Several limitations in this study should be acknowledged. First, it was a retrospective study based on public sequencing data, and the sample capacity of the tissue microarray verification cohort was insufficient. Hence, the prognostic and predictive efficacies of our CAFrelated signature should be prospectively verified in large clinical trial. In addition, cross-validations at proteomics level are also necessary to serve the clinical applications. Secondly, the molecular mechanisms of how these CAFrelated genes affect patient prognosis and therapeutic responses need to be clarified by further basic experimental studies.

\section{Conclusions}

In summary, based on integrated single-cell and bulk RNA sequencing analysis, we constructed and validated a nine-gene CAF-related signature as an independent prognostic indicator for CRC patients. Our results also provided genomic evidence for future research directions on anti-CAF therapeutic strategies for those who might not benefit from immunotherapy.

\footnotetext{
Abbreviations

CAF: Cancer-associated fibroblast; CRC: Colorectal cancer; scRNA-seq: Singlecell RNA sequencing; GEO: Gene Expression Omnibus; TCGA: The Cancer Genome Atlas; GDC: Genomic Data Commons; TME: Tumor microenvironment; ECM: Extracellular matrix; FAP: Fibroblast activation protein; ACTA2: Actin alpha 2; PDGFRB: Platelet derived growth factor receptor- $B ;$ CAV1: Caveolin 1; PDPN: Podoplanin; UMI: Unique molecular identifier; RMA: Robust Multi-array Average; READ: Rectal adenocarcinoma; COAD: Colon adenocarcinoma; t-SNE: T-distributed stochastic neighbor embedding; PCA: Principal component analysis; FC: Fold change; GO: Gene Ontology; KEGG: Kyoto Encyclopedia of Genes and Genomes; BP: Biological process; MF: Molecular function; CC: Cellular components; LASSO: Least absolute shrinkage operator; ssGSEA: Single sample gene set enrichment analysis; MCP-counter: Microenvironment Cell Populations-counter; EPIC: Estimate the Proportion of Immune and Cancer cells; IC50: Half-maximal inhibitory concentration; GDSC: Genomics of Drug Sensitivity in Cancer; TIDE: Tumor Immune Dysfunction and Exclusion; GSEA: Gene set enrichment analysis; MSigDB: The Molecular Signatures Database; C-index: Concordance index; HPA: Human Protein Atlas; IHC: Immunohistochemistry; DAB: Diaminobenzidine tetrachloride; $\mathrm{QPCR}$ : Real-time polymerase chain reaction; OS: Overall survival; HR: Hazard ratio; Cl: Confidence interval; PDGFRA: Platelet derived growth factor receptor alpha; ZEB1: Zinc finger E-box binding homeobox 1; FOXF1: Forkhead box F1; SPARC : Secreted protein acidic and cysteine rich; MMP2: Matrix metallopeptidase 2; FN1: Fibronectin 1; HSPB1: Heat shock protein family B (small) member 1; S100A13: S100 calcium binding protein A13; PPP1R14A: Protein phosphatase 1 regulatory inhibitor subunit 14A; CSRP2: Cysteine and glycine-rich protein 2; TPM2: Tropomyosin 2; CEBPD: CCAAT/enhancer binding protein; TIMP1: Tissue
}

inhibitor of metalloproteinases 1; SPINK1: Serine peptidase inhibitor Kazal type 1; CXCL1: C-X-C motif chemokine ligand 1; VEGF: Vascular endothelial growth factor.

\section{Supplementary Information}

The online version contains supplementary material available at https://doi. org/10.1186/s12935-021-02252-9.

Additional file 1: Table 1. qRT-PCR primer sequences.

Additional file 2: Figure S1. Validation analysis on single-cell RNA sequencing from 1771 cells of one CRC tissue (GSM3855015). (a) Post quality control filtering of each sequenced cell, which was plotted in violin plots to display their number of RNA features (nFeature_RNA) and absolute UMI counts (nCount_RNA). (b) Correlation analysis between nFeature and nCount. (c) Cells were clustered into 11 types via tSNE dimensionality reduction algorithm, each color represented the annotated phenotype of each cluster. (d) Heatmap depicting expressions of top 10 marker genes among 11 identified CRC cell clusters.

Additional file 3: Figure S2. (a) Recognized and (b) the identified CAF markers expressions in single-cell clusters of GSM3855015 sample. (c) Bubble plot visualizing genes expression characteristics in single-cell RNA sequencing profile. Cell phenotypes were listed on y-axis, recognized CAF markers (left part of the dotted line) as well as the identified 9 prognostic markers (right part of the dotted line) were listed along the $x$-axis. Dot size reflects each gene's expressing percentage of each cluster's cells; dot color represents the expression level.

\section{Acknowledgements}

Not applicable.

\section{Authors' contributions}

$\mathrm{HZ}$ and $\mathrm{XW}$ designed the research; $\mathrm{HZ}$ and $\mathrm{HSL}$ performed the bioinformatics analysis and interpreted the results; HSL and YG performed the experiments and analyzed the results; $\mathrm{HZ}$ drafted the manuscript; $Y G$ and $X W$ revised the manuscript and gave the final approval of the version to be published. All authors read and approved the final manuscript.

\section{Funding}

The study was not supported by any funding source.

\section{Availability of data and materials}

The datasets supporting the findings of this study are available in the from The Cancer Genome Atlas (TCGA) (https://gdc.xenahubs.net) and GEO (https:// www.ncbi.nlm.nih.gov/geo/2 databases. Further inquiries can be directed to the corresponding author.

\section{Declarations}

\section{Ethics approval and consent to participate}

The studies involving human participants were reviewed and approved by The Ethics Committee of Shanghai Outdo Biotech Co., Ltd and Peking University First Hospital. Written informed consent was obtained from all subjects in our study.

Consent for publication

Not applicable.

Competing interests

The authors declare that they have no competing interests.

Received: 17 July 2021 Accepted: 7 October 2021

Published online: 20 October 2021 


\section{References}

1. Bray F, Ferlay J, Soerjomataram I, Siegel RL, Torre LA, Jemal A. Global cancer statistics 2018: GLOBOCAN estimates of incidence and mortality worldwide for 36 cancers in 185 countries. CA Cancer J Clin. 2018:68(6):394-424

2. Siegel RL, Miller KD, Fuchs HE, Jemal A. Cancer statistics, 2021. CA Cancer J Clin. 2021;71(1):7-33

3. Van der Jeught K, Xu HC, Li YJ, Lu XB, Ji G. Drug resistance and new therapies in colorectal cancer. World J Gastroenterol. 2018;24(34):3834-48.

4. Shafabakhsh R, Arianfar F, Vosough M, Mirzaei HR, Mahjoubin-Tehran M, Khanbabaei $\mathrm{H}$, et al. Autophagy and gastrointestinal cancers: the behind the scenes role of long non-coding RNAs in initiation, progression, and treatment resistance. Cancer Gene Ther. 2021. https://doi.org/10.1038/ s41417-020-00272-7.

5. Pourhanifeh $\mathrm{MH}$, Vosough M, Mahjoubin-Tehran M, Hashemipour M, Nejati M, Abbasi-Kolli M, et al. Autophagy-related microRNAs: Possible regulatory roles and therapeutic potential in and gastrointestinal cancers. Pharmacol Res. 2020;161:105133.

6. Wang M, Zhao J, Zhang L, Wei F, Lian Y, Wu Y, et al. Role of tumor microenvironment in tumorigenesis. J Cancer. 2017;8(5):761-73.

7. Hanahan D, Coussens LM. Accessories to the crime: functions of cells recruited to the tumor microenvironment. Cancer Cell. 2012;21(3):309-22.

8. Crotti S, Piccoli M, Rizzolio F, Giordano A, Nitti D, Agostini M. Extracellular matrix and colorectal cancer: how surrounding microenvironment affects cancer cell behavior? J Cell Physiol. 2017;232(5):967-75.

9. Räsänen K, Vaheri A. Activation of fibroblasts in cancer stroma. Exp Cell Res. 2010;316(17):2713-22.

10. Swann JB, Smyth MJ. Immune surveillance of tumors. J Clin Investig. 2007;117(5):1137-46.

11. Nakagawa H, Liyanarachchi S, Davuluri RV, Auer H, Martin EW, de la Chapelle A, et al. Role of cancer-associated stromal fibroblasts in metastatic colon cancer to the liver and their expression profiles. Oncogene. 2004;23(44):7366-77.

12. Savardashtaki A, Shabaninejad Z, Movahedpour A, Sahebnasagh R, Mirzaei $\mathrm{H}$, Hamblin MR. miRNAs derived from cancer-associated fibroblasts in colorectal cancer. Epigenomics. 2019;11(14):1627-45.

13. Desmoulière A, Redard M, Darby I, Gabbiani G. Apoptosis mediates the decrease in cellularity during the transition between granulation tissue and scar. Am J Pathol. 1995;146(1):56-66

14. Haviv I, Polyak K, Qiu W, Hu M, Campbell I. Origin of carcinoma associated fibroblasts. Cell cycle (Georgetown, Tex). 2009;8(4):589-95.

15. I wano M, Plieth D, DanoffTM, Xue C, Okada H, Neilson EG. Evidence that fibroblasts derive from epithelium during tissue fibrosis. J Clin Investig. 2002;110(3):341-50.

16. Direkze NC, Hodivala-Dilke $K$, Jeffery R, Hunt T, Poulsom R, Oukrif D, et al. Bone marrow contribution to tumor-associated myofibroblasts and fibroblasts. Can Res. 2004;64(23):8492-5.

17. Russo FP, Alison MR, Bigger BW, Amofah E, Florou A, Amin F, et al. The bone marrow functionally contributes to liver fibrosis. Gastroenterology. 2006;130(6):1807-21.

18. Dvorak HF. Tumors: wounds that do not heal. N Engl J Med. 1986;315(26):1650-9.

19. Eyden B, Banerjee SS, Shenjere P, Fisher C. The myofibroblast and its tumours. J Clin Pathol. 2009;62(3):236-49.

20. Sahai E, Astsaturov I, Cukierman E, DeNardo DG, Egeblad M, Evans RM, et al. A framework for advancing our understanding of cancer-associated fibroblasts. Nat Rev Cancer. 2020;20(3):174-86.

21. Yamaguchi $H$, Sakai R. Direct interaction between carcinoma cells and cancer associated fibroblasts for the regulation of cancer invasion. Cancers (Basel). 2015;7(4):2054-62.

22. Razavi ZS, Asgarpour K, Mahjoubin-Tehran M, Rasouli S, Khan H, Shahrzad MK, et al. Angiogenesis-related non-coding RNAs and gastrointestinal cancer. Mol Ther Oncolytics. 2021;21:220-41.

23. Cirri P, Chiarugi P. Cancer-associated-fibroblasts and tumour cells: a diabolic liaison driving cancer progression. Cancer Metastasis Rev. 2012;31(1):195-208.

24. Sandberg TP, Stuart MPME, Oosting J, Tollenaar RAEM, Sier CFM, Mesker WE. Increased expression of cancer-associated fibroblast markers at the invasive front and its association with tumor-stroma ratio in colorectal cancer. BMC Cancer. 2019;19(1):284
25. Han C, Liu T, Yin R. Biomarkers for cancer-associated fibroblasts. Biomark Res. 2020;8(1):64

26. Togo S, Polanska UM, Horimoto Y, Orimo A. Carcinoma-associated fibroblasts are a promising therapeutic target. Cancers (Basel). 2013:5(1):149-69.

27. Park JE, Lenter MC, Zimmermann RN, Garin-Chesa P, Old LJ, Rettig WJ. Fibroblast activation protein, a dual specificity serine protease expressed in reactive human tumor stromal fibroblasts. J Biol Chem. 1999:274(51):36505-12.

28. Brennen WN, Isaacs JT, Denmeade SR. Rationale behind targeting fibroblast activation protein-expressing carcinoma-associated fibroblasts as a novel chemotherapeutic strategy. Mol Cancer Ther. 2012;11(2):257-66.

29. Kraman M, Bambrough PJ, Arnold JN, Roberts EW, Magiera L, Jones JO, et al. Suppression of antitumor immunity by stromal cells expressing fibroblast activation protein-alpha. Science (New York, NY). 2010;330(6005):827-30.

30. Teichgräber V, Monasterio C, Chaitanya K, Boger R, Gordon K, Dieterle $\mathrm{T}$, et al. Specific inhibition of fibroblast activation protein (FAP)-alpha prevents tumor progression in vitro. Adv Med Sci. 2015;60(2):264-72.

31. Hofheinz RD, Al-Batran SE, Hartmann F, Hartung G, Jäger D, Renner C, et al. Stromal antigen targeting by a humanised monoclonal antibody: an early phase II trial of sibrotuzumab in patients with metastatic colorectal cancer. Onkologie. 2003;26(1):44-8.

32. Narra K, Mullins SR, Lee HO, Strzemkowski-Brun B, Magalong K, Christiansen VJ, et al. Phase II trial of single agent Val-boroPro (Talabostat) inhibiting Fibroblast Activation Protein in patients with metastatic colorectal cancer. Cancer Biol Ther. 2007;6(11):1691-9.

33. Papalexi E, Satija R. Single-cell RNA sequencing to explore immune cell heterogeneity. Nat Rev Immunol. 2018;18(1):35-45.

34. Kim K-T, Lee HW, Lee H-O, Kim SC, Seo YJ, Chung W, et al. Single-cell mRNA sequencing identifies subclonal heterogeneity in anticancer drug responses of lung adenocarcinoma cells. Genome Biol. 2015;16(1):127.

35. Lee HO, Hong Y, Etlioglu HE, Cho YB, Pomella V, Van den Bosch B, et al. Lineage-dependent gene expression programs influence the immune landscape of colorectal cancer. Nat Genet. 2020;52(6):594-603.

36. Goldman MJ, Craft B, Hastie M, Repečka K, McDade F, Kamath A, et al. Visualizing and interpreting cancer genomics data via the Xena platform. Nat Biotechnol. 2020;38(6):675-8.

37. Leek JT, Johnson WE, Parker HS, Jaffe AE, Storey JD. The sva package for removing batch effects and other unwanted variation in high-throughput experiments. Bioinformatics. 2012;28(6):882-3.

38. Stuart T, Butler A, Hoffman P, Hafemeister C, Papalexi E, Mauck WM III, et al. Comprehensive integration of single-cell data. Cell. 2019;177(7):1888-902. e21.

39. Hinton G. Visualizing high-dimensional data using t-SNE. Vigiliae Christianae. 2008;9:2579-605.

40. Aran D, Looney AP, Liu L, Wu E, Fong V, Hsu A, et al. Reference-based analysis of lung single-cell sequencing reveals a transitional profibrotic macrophage. Nat Immunol. 2019;20(2):163-72.

41. Yu G, Wang L-G, Han Y, He Q-Y. clusterProfiler: an R package for comparing biological themes among gene clusters. OMICS. 2012;16(5):284-7.

42. Simon N, Friedman J, Hastie T, Tibshirani R. Regularization paths for Cox's proportional hazards model via coordinate descent. J Stat Softw. 2011;39(5):1-13.

43. Hänzelmann S, Castelo R, Guinney J. GSVA: gene set variation analysis for microarray and RNA-Seq data. BMC Bioinformatics. 2013;14(1):7.

44. Bindea G, Mlecnik B, Tosolini M, Kirilovsky A, Waldner M, Obenauf Anna C, et al. Spatiotemporal dynamics of intratumoral immune cells reveal the immune landscape in human cancer. Immunity. 2013;39(4):782-95.

45. Becht E, Giraldo NA, Lacroix L, Buttard B, Elarouci N, Petitprez F, et al. Estimating the population abundance of tissue-infiltrating immune and stromal cell populations using gene expression. Genome Biol. 2016;17(1):218

46. Racle J, de Jonge K, Baumgaertner P, Speiser DE, Gfeller D. Simultaneous enumeration of cancer and immune cell types from bulk tumor gene expression data. Elife. 2017;6:e26476.

47. Sturm G, Finotello F, List M. Immunedeconv: an R Package for unified access to computational methods for estimating immune cell fractions from bulk RNA-Sequencing data. In: Boegel S, editor. Bioinformatics for 
cancer immunotherapy: methods and protocols. Springer, US: New York, NY; 2020. p. 223-32.

48. Yoshihara K, Shahmoradgoli M, Martínez E, Vegesna R, Kim H, TorresGarcia W, et al. Inferring tumour purity and stromal and immune cell admixture from expression data. Nat Commun. 2013;4:2612.

49. Geeleher P, Cox NJ, Huang RS. Clinical drug response can be predicted using baseline gene expression levels and in vitrodrug sensitivity in cell lines. Genome Biol. 2014;15(3):R47.

50. Geeleher P, Cox N, Huang RS. pRRophetic: An R package for prediction of clinical chemotherapeutic response from tumor gene expression levels. PLOS ONE. 2014;9(9):e107468.

51. Yang W, Soares J, Greninger P, Edelman EJ, Lightfoot H, Forbes S, et al. Genomics of Drug Sensitivity in Cancer (GDSC): a resource for therapeutic biomarker discovery in cancer cells. Nucleic Acids Res. 2013;41(D1):D955-61.

52. Jiang P, Gu S, Pan D, Fu J, Sahu A, Hu X, et al. Signatures of T cell dysfunction and exclusion predict cancer immunotherapy response. Nat Med. 2018;24(10):1550-8.

53. Liberzon A, Birger C, Thorvaldsdóttir H, Ghandi M, Mesirov JP, Tamayo P. The Molecular Signatures Database (MSigDB) hallmark gene set collection. Cell Syst. 2015;1(6):417-25.

54. Sergushichev AA. An algorithm for fast preranked gene set enrichment analysis using cumulative statistic calculation. bioRxiv. 2016:060012.

55. Uhlén M, Fagerberg L, Hallström BM, Lindskog C, Oksvold P, Mardinoglu A, et al. Proteomics. Tissue-based map of the human proteome. Science (New York, NY). 2015;347(6220):1260419.

56. Gascard P, TIsty TD. Carcinoma-associated fibroblasts: orchestrating the composition of malignancy. Genes Dev. 2016;30(9):1002-19.

57. Loeffler M, Krüger JA, Niethammer AG, Reisfeld RA. Targeting tumorassociated fibroblasts improves cancer chemotherapy by increasing intratumoral drug uptake. J Clin Investig. 2006;116(7):1955-62.

58. Kalluri R. The biology and function of fibroblasts in cancer. Nat Rev Cancer. 2016;16(9):582-98.

59. Tumeh PC, Harview CL, Yearley JH, Shintaku IP, Taylor EJ, Robert L, et al. PD-1 blockade induces responses by inhibiting adaptive immune resistance. Nature. 2014;515(7528):568-71.

60. Ford K, Hanley CJ, Mellone M, Szyndralewiez C, Heitz F, Wiesel P, et al. NOX4 inhibition potentiates immunotherapy by overcoming cancerassociated fibroblast-mediated CD8 T-cell exclusion from tumors. Can Res. 2020;80(9):1846.

61. Cukierman E, Bassi DE. Physico-mechanical aspects of extracellular matrix influences on tumorigenic behaviors. Semin Cancer Biol. 2010;20(3):139-45.

62. Yu W-D, Sun G, Li J, Xu J, Wang X. Mechanisms and therapeutic potentials of cancer immunotherapy in combination with radiotherapy and/or chemotherapy. Cancer Lett. 2019;452:66-70.

63. McDonnell AM, Lesterhuis WJ, Khong A, Nowak AK, Lake RA, Currie AJ, et al. Tumor-infiltrating dendritic cells exhibit defective cross-presentation of tumor antigens, but is reversed by chemotherapy. Eur J Immunol. 2015;45(1):49-59.

64. Sarvizadeh M, Hasanpour O, Naderi Ghale-Noie Z, Mollazadeh S, Rezaei $\mathrm{M}$, Pourghadamyari $\mathrm{H}$, et al. Allicin and digestive system cancers: from chemical structure to its therapeutic opportunities. Front Oncol. 2021;11:650256.

65. Ashrafizadeh M, Zarrabi A, Hashemipour M, Vosough M, Najafi M, Shahinozzaman $M$, et al. Sensing the scent of death: modulation of microRNAs by Curcumin in gastrointestinal cancers. Pharmacol Res. 2020;160:105199.

66. Dong J, Ma Q. TIMP1 promotes multi-walled carbon nanotube-induced lung fibrosis by stimulating fibroblast activation and proliferation. Nanotoxicology. 2017;11(1):41-51.

67. Bourboulia D, Stetler-Stevenson WG. Matrix metalloproteinases (MMPs) and tissue inhibitors of metalloproteinases (TIMPs): positive and negative regulators in tumor cell adhesion. Semin Cancer Biol. 2010;20(3):161-8.

68. Alpízar-Alpízar W, Laerum OD, Christensen IJ, Ovrebo K, Skarstein A, Høyer-Hansen G, et al. Tissue inhibitor of Metalloproteinase-1 Is confined to tumor-associated myofibroblasts and is increased with progression in gastric adenocarcinoma. J Histochem Cytochem. 2016;64(8):483-94.

69. Illemann M, Eefsen RHL, Bird NC, Majeed A, Osterlind K, Laerum OD, et al. Tissue inhibitor of matrix metalloproteinase-1 expression in colorectal cancer liver metastases is associated with vascular structures. Mol Carcinog. 2016;55(2):193-208.

70. Gong Y, Scott E, Lu R, Xu Y, Oh WK, Yu Q. TIMP-1 promotes accumulation of cancer associated fibroblasts and cancer progression. PLOS ONE. 2013:8(10):e77366-e.

71. Pape J, Magdeldin T, Stamati K, Nyga A, Loizidou M, Emberton M, et al. Cancer-associated fibroblasts mediate cancer progression and remodel the tumouroid stroma. Br J Cancer. 2020;123(7):1178-90.

72. Song $G$, Xu S, Zhang $H$, Wang $Y$, Xiao C, Jiang T, et al. TIMP1 is a prognostic marker for the progression and metastasis of colon cancer through FAK-PI3K/AKT and MAPK pathway. J Exp Clin Cancer Res. 2016;35(1):148

73. Koskensalo S, Hagström J, Louhimo J, Stenman UH, Haglund C. Tumourassociated trypsin inhibitor TATI is a prognostic marker in colorectal cancer. Oncology. 2012;82(4):234-41.

74. Koskensalo S, Louhimo J, Hagström J, Lundin M, Stenman U-H, Haglund C. Concomitant tumor expression of EGFR and TATI/SPINK1 associates with better prognosis in colorectal cancer. PLoS ONE. 2013;8(10):e76906-e.

75. Chen YT, Tsao SC, Tsai HP, Wang JY, Chai CY. Serine protease inhibitor Kazal type 1 (SPINK1) as a prognostic marker in stage IV colon cancer patients receiving cetuximab based targeted therapy. J Clin Pathol. 2016;69(11):974-8.

76. Ida S, Ozaki N, Araki K, Hirashima K, Zaitsu Y, Taki K, et al. SPINK1 status in colorectal cancer, impact on proliferation, and role in colitis-associated cancer. Mol Cancer Res MCR. 2015;13(7):1130-8.

77. Tiwari R, Pandey SK, Goel S, Bhatia V, Shukla S, Jing X, et al. SPINK1 promotes colorectal cancer progression by downregulating Metallothioneins expression. Oncogenesis. 2015;4(8):e162.

78. Liu X, Bing Z, Wu J, Zhang J, Zhou W, Ni M, et al. Integrative gene expression profiling analysis to investigate potential prognostic biomarkers for colorectal cancer. Medical Sci Monit Int Med J Exp Clin Res. 2020:26:e918906.

79. Zhuo C, Wu X, Li J, Hu D, Jian J, Chen C, et al. Chemokine (C-X-C motif) ligand 1 is associated with tumor progression and poor prognosis in patients with colorectal cancer. 2018. Biosci Rep. https://doi.org/10.1042/ BSR20180580.

80. Oladipo O, Conlon S, O'Grady A, Purcell C, Wilson C, Maxwell PJ, et al. The expression and prognostic impact of CXC-chemokines in stage II and III colorectal cancer epithelial and stromal tissue. Br J Cancer. 2011;104(3):480-7.

81. le Rolle AF, Chiu TK, Fara M, Shia J, Zeng Z, Weiser MR, et al. The prognostic significance of CXCL1 hypersecretion by human colorectal cancer epithelia and myofibroblasts. J TransI Med. 2015;13:199.

82. Riecken LB, Zoch A, WiehI U, Reichert S, Scholl I, Cui Y, et al. CPI-17 drives oncogenic Ras signaling in human melanomas via Ezrin-Radixin-Moesin family proteins. Oncotarget. 2016;7(48):78242-54.

83. Jin H, Sperka T, Herrlich P, Morrison H. Tumorigenic transformation by $\mathrm{CPI}-17$ through inhibition of a merlin phosphatase. Nature. 2006;442(7102):576-9.

84. Li D, Guo J, Wang S, Zhu L, Shen Z. Identification of novel methylated targets in colorectal cancer by microarray analysis and construction of co-expression network. Oncol Lett. 2017;14(3):2643-8.

85. Ali D, Honne H, Danielsen SA, Cekaite L, Meling Gl, Rognum TO, et al. 694 Identification of novel epigenetic biomarkers in colorectal cancer, GLDC and PPP1R14A. Eur J Cancer Suppl. 2010;8(5):175.

86. Zhou Y, Bian S, Zhou X, Cui Y, Wang W, Wen L, et al. Single-cell multiomics sequencing reveals prevalent genomic alterations in tumor stromal cells of human colorectal cancer. Cancer Cell. 2020;38(6):818-28.e5.

87. Wang J, Guan X, Zhang Y, Ge S, Zhang L, Li H, et al. Exosomal miR27a derived from gastric cancer cells regulates the transformation of fibroblasts into cancer-associated fibroblasts. Cell Physiol Biochem. 2018;49(3):869-83.

88. Chi J-Y, Hsiao Y-W, Li C-F, Lo Y-C, Lin Z-Y, Hong J-Y, et al. Targeting chemotherapy-induced PTX3 in tumor stroma to prevent the progression of drug-resistant cancers. Oncotarget. 2015;6(27):23987-4001.

89. Wang D, Cheng X, Li Y, Guo M, Zhao W, Qiu J, et al. C/EBPS-Slug-Lox1 axis promotes metastasis of lung adenocarcinoma via oxLDL uptake. Oncogene. 2020;39(4):833-48. 
90. Marenholz I, Heizmann CW, Fritz G. S100 proteins in mouse and man: from evolution to function and pathology (including an update of the nomenclature). Biochem Biophys Res Commun. 2004;322(4):1111-22.

91. Al-Mutairy EA, Imtiaz FA, Khalid M, Al Qattan S, Saleh S, Mahmoud LM, et al. An atypical pulmonary fibrosis is associated with co-inheritance of mutations in the calcium binding protein genes S100A3 and S100A13. Eur Respir J. 2019;54(1):1802041

92. Massi D, Landriscina M, Piscazzi A, Cosci E, Kirov A, Paglierani M, et al. S100A13 is a new angiogenic marker in human melanoma. Mod Pathol. 2010;23(6):804-13.

93. Landriscina M, Schinzari G, Di Leonardo G, Quirino M, Cassano A, D'Argento E, et al. S100A13, a new marker of angiogenesis in human astrocytic gliomas. J Neurooncol. 2006;80(3):251-9.
94. Lee Y-J, Lee H-J, Choi S-H, Jin YB, An HJ, Kang J-H, et al. Soluble HSPB1 regulates VEGF-mediated angiogenesis through their direct interaction. Angiogenesis. 2012;15(2):229-42.

\section{Publisher's Note}

Springer Nature remains neutral with regard to jurisdictional claims in published maps and institutional affiliations.
Ready to submit your research? Choose BMC and benefit from:

- fast, convenient online submission

- thorough peer review by experienced researchers in your field

- rapid publication on acceptance

- support for research data, including large and complex data types

- gold Open Access which fosters wider collaboration and increased citations

- maximum visibility for your research: over $100 \mathrm{M}$ website views per year

At BMC, research is always in progress.

Learn more biomedcentral.com/submissions 